hep-th/0403230

SNB/March/2004

\title{
Geometrical Aspects of BRST Cohomology in Augmented Superfield Formalism
}

\author{
R.P.Malik * \\ S. N. Bose National Centre for Basic Sciences, \\ Block-JD, Sector-III, Salt Lake, Calcutta-700 098, India
}

\begin{abstract}
In the framework of augmented superfield approach, we provide the geometrical origin and interpretation for the nilpotent (anti-)BRST charges, (anti-)co-BRST charges and a non-nilpotent bosonic charge. Together, these local and conserved charges turn out to be responsible for a clear and cogent definition of the Hodge decomposition theorem in the quantum Hilbert space of states. The above charges owe their origin to the de Rham cohomological operators of differential geometry which are found to be at the heart of some of the key concepts associated with the interacting gauge theories. For our present review, we choose the two $(1+1)$-dimensional (2D) quantum electrodynamics (QED) as a prototype field theoretical model to derive all the nilpotent symmetries for all the fields present in this interacting gauge theory in the framework of augmented superfield formulation and show that this theory is a unique example of an interacting gauge theory which provides a tractable field theoretical model for the Hodge theory.
\end{abstract}

PACS: 11.15.-q; 12.20.-m; 11.30.Ph; 02.20.+b

Keywords: Augmented superfield formalism; (co-)BRST symmetries; (dual-)horizontality conditions; QED in two-dimensions; Hodge decomposition theorem

\footnotetext{
*E-mail address: malik@boson.bose.res.in
} 


\section{Introduction}

One of the most elegant and intuitive approaches to covariantly quantize a gauge theory, endowed with the first-class constraints [1] in the language of the Dirac's classification scheme, is the Becchi-Rouet-Stora-Tyutin (BRST) formalism (see, e.g, [2]). In this formalism, the unitarity and "quantum" gauge (i.e. BRST) invariance are respected together at any arbitrary order of perturbative computations connected with the physical processes involving the gauge fields and the matter fields of any arbitrary interacting 1-form (non-)Abelian gauge theories (see, e.g. $[3,4])^{\dagger}$. The BRST formalism is indispensable in the context of the modern developments in the topological field theories and topological string theories [57]. The range of the applicability of this formalism has been extended by the inclusion of the second-class constraints in its ever widening framework (see, e.g., [8]). Its deep connections with the mathematics of cohomology and differential geometry, its beautiful interpretation in the framework of superfield formulation, its intimate relationships with the basic tenets of supersymmetry, its fruitful application in the context of reparametrization invariant theories such as supergravity theories, superstring theories, brane dynamics, M-theory, etc., have elevated this area of research to a high degree of mathematical sophistication as well as physical applications (see, e.g., [9,10] and references therein).

The geometrical aspects of any arbitrary physical theory provide a clear physical interpretation for the key theoretical ideas expressed in their abstract mathematical form. One of the most attractive, accurate and intuitive theoretical approaches to gain an insight into the geometrical origin of the existence of the nilpotent (anti-)BRST symmetry transformations (and their corresponding conserved and nilpotent generators), for any arbitrary $p$-form (non-)Abelian gauge theories, is the superfield formalism [11-18]. In this formalism, a $(p+1)$-form super curvature $\tilde{F}=\tilde{d} \tilde{A}+\tilde{A} \wedge \tilde{A}$ is constructed from the super exterior derivative $\tilde{d}=d x^{\mu} \partial_{\mu}+d \theta \partial_{\theta}+d \bar{\theta} \partial_{\bar{\theta}}$ (with $\tilde{d}^{2}=0$ ) and a super $p$-form $(p=1,2,3, \ldots .$.$) connection \tilde{A}$ on a $(D+2)$-dimensional supermanifold parametrized by the superspace variables $Z^{M}=\left(x^{\mu}, \theta, \bar{\theta}\right)$ where $x^{\mu}(\mu=0,1,2 \ldots \ldots D-1)$ are the $D$-number of even (bosonic) spacetime coordinates and $\theta$ and $\bar{\theta}$ are a pair of Grassmannian (odd) variables (i.e. $\left.\theta^{2}=\bar{\theta}^{2}=0, \theta \bar{\theta}+\bar{\theta} \theta=0\right)$. This $(p+1)$-form super curvature is subsequently equated, due to the so-called horizontality condition ${ }^{\ddagger}$, with the $(p+1)$-form ordinary curvature $F=d A+A \wedge A$ defined on the ordinary $D$-dimensional flat Minkowski manifold with the help of an ordinary exterior derivative $d=d x^{\mu} \partial_{\mu}$ (with $d^{2}=0$ ) and a $p$-form ordinary connection $A$. This condition leads to the derivation of the nilpotent (anti-)BRST symmetry transformations for the gauge field and the (anti-)ghost fields of the $p$-form gauge theory. We christen this superfield formulation as the usual superfield

${ }^{\dagger}$ The full strength of the BRST formalism turns up in its full glory in the context of non-Abelian gauge theories where, for each loop diagram consisting of the gauge (gluon) fields alone, there exists a diagram consisting of the (anti-)ghost fields as its counterpart so that the unitarity in the theory can be maintained at any arbitrary order of perturbative computation (see, e.g., [3] for detailed computations).

${ }_{\ddagger}^{\ddagger}$ This condition is referred to as the soul-flatness condition by Nakanishi and Ojima [19]. 
approach because, as is evident, this formalism does not shed any light on the derivation of the nilpotent (anti-)BRST symmetry transformations for the matter fields of an interacting gauge theory (where there is a coupling between the gauge field and matter fields). However, this approach does provide the geometrical origin and interpretation for (i) the (anti-)BRST symmetry transformations and their corresponding generators as the translation generators $\left(\operatorname{Lim}_{\bar{\theta} \rightarrow 0}(\partial / \partial \theta), \operatorname{Lim}_{\theta \rightarrow 0}(\partial / \partial \bar{\theta})\right)$ along the Grassmannian directions of the $(D+2)$-dimensional supermanifold, (ii) the nilpotency property associated with the (anti)BRST symmetry transformations (and corresponding conserved and nilpotent generators) as a couple of successive translations (i.e. $\left.(\partial / \partial \theta)^{2}=(\partial / \partial \bar{\theta})^{2}=0\right)$ along either the $\theta$ or $\bar{\theta}$-directions of the supermanifold, and (iii) the anti-commutativity property of the local, covariant, continuous and nilpotent BRST and anti-BRST symmetry transformations (and corresponding conserved and nilpotent (anti-)BRST charges) as a couple of translations along the $(\theta)$ - and $(\bar{\theta})$-directions followed by another couple of translations along the Grassmannian directions with the reverse order (i.e. $(\partial / \partial \theta)(\partial / \partial \bar{\theta})+(\partial / \partial \bar{\theta})(\partial / \partial \theta)=0)$.

It is obvious from the above discussions that only one (i.e. $\tilde{d}$ ) of the three ${ }^{\S}$ super de Rham cohomological operators $(\tilde{d}, \tilde{\delta}, \tilde{\Delta})$ has been exploited in the horizontality condition that provides the geometrical interpretation for the conserved and nilpotent (anti-)BRST charges which generate the local, covariant, continuous and nilpotent symmetries for the gauge theories. In the usual discussions on the BRST cohomology, only the nilpotent BRST charge (i.e. the analogue of the cohomological operator $d$ ) plays the central role. However, a thorough discussion on the BRST cohomology requires the existence of a nilpotent coBRST charge (i.e. the analogue of the cohomological operator $\delta= \pm * d *$ ) so that the celebrated Hodge decomposition theorem can be defined precisely. Towards accomplishing this goal, in a recent set of papers [25-28], all the super de Rham cohomological operators have been exploited in the framework of the usual superfield formulation to derive the local, covariant and continuous (anti-)BRST, (anti-)co-BRST and a bosonic symmetry transformations for the gauge- and (anti-)ghost fields of the two $(1+1)$-dimensional free (non-)Abelian gauge theories defined on a four $(2+2)$-dimensional supermanifold. In these derivations, the generalized versions of the horizontality condition play very decisive roles. The geometrical interpretations for all the above symmetries have been provided in the framework of the usual superfield formulation. Interestingly, for the first time, the geometrical meaning of the topological nature of the above field theories has been provided in the framework of usual superfield formulation where it has been shown that

\footnotetext{
${ }^{\S}$ On an ordinary manifold without a boundary, the set of operators $(d, \delta, \Delta)$ define the de Rham cohomological operators of the differential geometry. The (co-)exterior derivatives $(\delta) d$ and the Laplacian operator $\Delta=d \delta+\delta d$ obey an algebra: $d^{2}=\delta^{2}=0, \Delta=(d+\delta)^{2},[\Delta, d]=0,[\Delta, \delta]=0$ where $d=d x^{\mu} \partial_{\mu}$ and $\delta= \pm * d *$ are connected with each-other by the Hodge duality $(*)$ operation defined on the manifold and the $(+)-$ signs (present in $\delta= \pm * d *$ ) depend on (i) the degree of the differential forms involved in the inner product, and (ii) the dimensionality of the manifold. The Hodge decomposition (i.e. $f_{n}=\omega_{n}+d e_{n-1}+\delta c_{n+1}$ ) of an arbitrary differential form $f_{n}$ (of degree $n$ ) owes its origin to the above cohomological operators that define $\omega_{n}$ as the harmonic form (i.e. $\left.d \omega_{n}=\delta \omega_{n}=0\right), d e_{n-1}$ as the exact form and $\delta c_{n+1}$ as the co-exact form into which the arbitrary form $f_{n}$ is decomposed on the manifold (see, e.g. [20-24] for details).
} 
the Lagrangian density and the symmetric energy momentum tensor for these theories can be interpreted as the translation of some local (but composite) superfields along the Grassmannian directions of the supermanifold [29,30]. However, in the above attempts [2530] too, there is no discussion on the derivations of the above symmetry transformations for the matter fields present in an interacting gauge theory (which is more natural than the free gauge theories). Thus, the results of the usual superfield approach [11-18] (as well as its extended version [25-30]) are still incomplete as far as the derivation of all the nilpotent transformations for all the fields present in an interacting gauge theory is concerned.

In our recent set of papers [31-34], the above superfield formulations, endowed with the generalized versions of the horizontality condition, have been augmented by the additional restrictions that owe their origin to the requirement of the invariance of the matter conserved currents on the (super-)manifolds. We christen this version of the superfield approach as the augmented superfield formalism because it sheds light on the derivation of the nilpotent symmetries for all the fields (including the matter fields) present in an interacting gauge theory. The purpose of the present paper is to derive all the off-shell as well as the on-shell nilpotent (anti-)BRST and (anti-)co-BRST symmetry transformations for all the fields present in the BRST invariant Lagrangian density (cf. (2.1),(2.2) below) for the two $(1+1)$-dimensional interacting gauge theory (i.e. 2D QED $₫$ ) in the framework of augmented superfield formalism. We show that the off-shell nilpotent (anti-)BRST as well as (anti-)co-BRST symmetry transformations (and their corresponding nilpotent generators) correspond to the translation generators $\left(\operatorname{Lim}_{\bar{\theta} \rightarrow 0}(\partial / \partial \theta), \operatorname{Lim}_{\theta \rightarrow 0}(\partial / \partial \bar{\theta})\right)$ along the Grassmannian $(\theta) \bar{\theta}$-directions of the four $(2+2)$-dimensional supermanifold. The process of translation of the general superfields produces the internal (anti-)BRST and (anti-)coBRST symmetry transformations on their counterparts ordinary fields, defined on the ordinary Minkowski flat manifold. We also demonstrate that the on-shell nilpotent (anti-)BRST and (anti-)co-BRST symmetry transformations owe their origin to (anti-)chiral superfields where the on-shell nilpotent conserved charges (and their corresponding symmetries) are interpreted as the translation generators $((\partial / \partial \theta),(\partial / \partial \bar{\theta}))$ along a specific Grassmannian direction (i.e. $\theta$ or $\bar{\theta}$ ) of the three $(2+1)$-dimensional (anti-)chiral super sub-manifold of the most general four $(2+2)$-dimensional supermanifold. We have chosen, for our discussions, the 2D QED as a prototype example of an interacting gauge theory primarily for three reasons. First, the dual(co-)BRST transformations for this theory are local, covariant, continuous and nilpotent $[35,36]$. This is in sheer contrast with the interacting 4D Abelian gauge theory where the corresponding transformations are non-local, non-covariant, continuous and nilpotent (see, e.g., [37,38]). Second, it is a unique interacting gauge theory which provides a tractable field theoretical model for the Hodge theory (see, e.g., [36]). The other set of field theoretical models, that have been shown to be the examples of the Hodge theory, are (i) the free 2D Abelian 1-from gauge theory [39-41], (ii) the self-interacting 2D non-Abelian 1-form gauge theory (without any interaction with matter fields) [41,42], and

"A dynamically closed system of the photon field and the Dirac fields in 2D. 
(iii) the 4D free Abelian 2-form gauge theory [43,44]. It should be noted that there exist no interaction terms (involving the gauge field and matter fields) in the field theoretical models cited in (i), (ii) and (iii). Finally, 2D QED is a field theoretical model where the topological gauge field $A_{\mu}$ (see, e.g., [41] for details) couples with the matter conserved current $J_{\mu} \sim \bar{\psi} \gamma_{\mu} \psi$. Thus, 2D QED is interesting by itself because it is a very specific model for the interacting topological field theory $[35,36]$.

The material of our present paper is organized as follows. In Section 2, we recapitulate the bare essentials of our earlier investigations $[35,36]$ on the existence of the on-shell as well as off-shell nilpotent symmetries, a bosonic symmetry and a ghost (scale) symmetry transformations for the 2D interacting gauge theory (i.e. QED) in the framework of Lagrangian formulation. Section 3 is devoted to the derivation of the on-shell as well off-shell nilpotent symmetries for the gauge- and (anti-)ghost fields in the framework of usual superfield formulation. In Section 4, we deal with a detailed discussion (and an accurate derivation) of the nilpotent symmetries for the matter (Dirac) fields of this interacting gauge theory in the framework of augmented superfield formalism. Finally, in Section 5, we make some concluding remarks and indicate briefly the future perspective of our present endeavour.

\section{Preliminary: (Anti-)BRST- and (Anti-)co-BRST Symmetries}

We briefly demonstrate here the existence of the nilpotent (anti-)BRST and (anti-)coBRST symmetry transformations for the interacting two $(1+1)$-dimensional (2D) QED in the framework of Lagrangian formulation [36]. To this end in mind, we begin with the following Lagrangian density for the above interacting physical system in the Feynman gauge $\|[4,19,45,46]$

$$
\begin{aligned}
\mathcal{L}_{b} & =-\frac{1}{4} F^{\mu \nu} F_{\mu \nu}+\bar{\psi}\left(i \gamma^{\mu} D_{\mu}-m\right) \psi+B(\partial \cdot A)+\frac{1}{2} B^{2}-i \partial_{\mu} \bar{C} \partial^{\mu} C \\
& \equiv \frac{1}{2} E^{2}+\bar{\psi}\left(i \gamma^{\mu} D_{\mu}-m\right) \psi+B(\partial \cdot A)+\frac{1}{2} B^{2}-i \partial_{\mu} \bar{C} \partial^{\mu} C
\end{aligned}
$$

where $F_{\mu \nu}=\partial_{\mu} A_{\nu}-\partial_{\nu} A_{\mu}$ is the field strength tensor for the $U(1)$ gauge theory that is derived from the 2-form $d A=\frac{1}{2}\left(d x^{\mu} \wedge d x^{\nu}\right) F_{\mu \nu}$. As is evident, the latter is constructed by the application of the exterior derivative $d=d x^{\mu} \partial_{\mu}$ (with $d^{2}=0$ ) on the 1-form $A=d x^{\mu} A_{\mu}$ (which defines the vector potential $A_{\mu}$ ). It will be noted that in $2 \mathrm{D}, F_{\mu \nu}$ has only the electric component (i.e. $F_{01}=E$ ) and there is no magnetic component associated with it. The gauge-fixing term $\left(\partial \cdot A=\partial_{\mu} A^{\mu} \equiv \partial_{0} A_{0}-\partial_{1} A_{1}\right)$ is derived through the operation of the co-exterior derivative $\delta$ (with $\delta=-* d *, \delta^{2}=0$ ) on the one-form $A$ (i.e. $\delta A=-* d * A=(\partial \cdot A))$ where $*$ is the Hodge duality operation. The operation of the Laplacian operator $\Delta=d \delta+\delta d$ on the 1 -form $A=d x^{\mu} A_{\mu}$ leads to $\Delta A=d x^{\mu} \square A_{\mu}$. This,

"We adopt here the conventions and notations such that the flat $2 \mathrm{D}$ Minkowski metric is $\eta_{\mu \nu}=\operatorname{diag}$ $(+1,-1)$ and $\square=\eta^{\mu \nu} \partial_{\mu} \partial_{\nu}=\left(\partial_{0}\right)^{2}-\left(\partial_{1}\right)^{2}, \varepsilon_{01}=\varepsilon^{10}=+1, F_{01}=-\varepsilon^{\mu \nu} \partial_{\mu} A_{\nu}=F^{10}=\partial_{o} A_{1}-\partial_{1} A_{0}, D_{\mu} \psi=$ $\partial_{\mu} \psi+i e A_{\mu} \psi$. The Dirac matrices in the two-dimensional spacetime are: $\gamma^{0}=\sigma_{2}, \gamma^{1}=i \sigma_{1}, \gamma_{5}=\gamma^{0} \gamma^{1}=$ $\sigma_{3},\left\{\gamma^{\mu}, \gamma^{\nu}\right\}=2 \eta^{\mu \nu}, \gamma_{\mu} \gamma_{5}=\varepsilon_{\mu \nu} \gamma^{\nu}$. Here the Greek indices $\mu, \nu, \rho \ldots \ldots=0,1$ stand for the spacetime directions on the the 2D Minkowski manifold and $\sigma$ 's are the usual $2 \times 2$ Pauli matrices. 
in turn, produces the equation of motion for the gauge field $A_{\mu}$, present in the gauge-fixed Lagrangian density, if we demand that the Laplace equation $\Delta A=-e J$ (with a 1-form source term $J=d x^{\mu} J_{\mu}$ ) to be satisfied. Here $J_{\mu}=\bar{\psi} \gamma_{\mu} \psi$ is constructed by the Dirac fields $\psi$ and $\bar{\psi}$. Two important points to be noted, at this juncture, are (i) the kinetic energy and gauge-fixing terms of the Lagrangian density (2.1) owe their origin to the two (i.e. $d$ and $\delta$ ) of the three de Rham cohomological operators (i.e. $d, \delta, \Delta$ ) of the differential geometry. (ii) The Laplacian operator $\Delta=d \delta+\delta d$ produces the equation of motion (i.e. $\square A_{\mu}=-e J_{\mu}$ ) that results in from the the above gauge-fixed Lagrangian density. In the above Lagrangian density, the fermionic Dirac fields $(\psi, \bar{\psi})$, with the mass $m$ and charge $e$, couple to the $U(1)$ gauge field $A_{\mu}$ (i.e. $\left.-e \bar{\psi} \gamma^{\mu} A_{\mu} \psi\right)$ through the conserved current $J_{\mu}=\bar{\psi} \gamma_{\mu} \psi$. The anticommuting $\left(C \bar{C}+\bar{C} C=0, C^{2}=\bar{C}^{2}=0, C \psi+\psi C=0, \bar{\psi} C+C \bar{\psi}=0\right.$, etc.,) (anti-)ghost fields $(\bar{C}) C$ are required to maintain the unitarity and "quantum" gauge (i.e. BRST) invariance together at any arbitrary order of perturbation theory. The kinetic energy term $\left(\frac{1}{2} E^{2}\right)$ of $(2.1)$ can be linearized by invoking an additional auxiliary field $\mathcal{B}$

$$
\mathcal{L}_{B}=\mathcal{B} E-\frac{1}{2} \mathcal{B}^{2}+\bar{\psi}\left(i \gamma^{\mu} D_{\mu}-m\right) \psi+B(\partial \cdot A)+\frac{1}{2} B^{2}-i \partial_{\mu} \bar{C} \partial^{\mu} C,
$$

which is the analogue of the Nakanishi-Lautrup auxiliary field $B$ that is required to linearize the gauge-fixing term $-\frac{1}{2}(\partial \cdot A)^{2}$ in $(2.1)$. The above Lagrangian density $(2.2)$ respects the following off-shell nilpotent $\left(s_{(a) b}^{2}=0, s_{(a) d}^{2}=0\right)\left(\right.$ anti-)BRST $\left(s_{(a) b}\right)^{* *}$ - and (anti-)dual(co)$\operatorname{BRST}\left(s_{(a) d}\right)$ symmetry transformations (with $s_{b} s_{a b}+s_{a b} s_{b}=0, s_{d} s_{a d}+s_{a d} s_{d}=0$ ) [36]

$$
\begin{aligned}
& s_{b} A_{\mu}=\partial_{\mu} C, \quad s_{b} C=0, \quad s_{b} \bar{C}=i B, \quad s_{b} \psi=-i e C \psi, \\
& s_{b} \bar{\psi}=-i e \bar{\psi} C, \quad s_{b} \mathcal{B}=0, \quad s_{b} B=0, \quad s_{b} E=0, \quad s_{b}(\partial \cdot A)=\square C, \\
& s_{a b} A_{\mu}=\partial_{\mu} \bar{C}, \quad s_{a b} \bar{C}=0, \quad s_{a b} C=-i B, \quad s_{a b} \psi=-i e \bar{C} \psi, \\
& s_{a b} \bar{\psi}=-i e \bar{\psi} \bar{C}, \quad s_{a b} \mathcal{B}=0, \quad s_{a b} B=0, \quad s_{a b} E=0, \quad s_{a b}(\partial \cdot A)=\square \bar{C}, \\
& s_{d} A_{\mu}=-\varepsilon_{\mu \nu} \partial^{\nu} \bar{C}, \quad s_{d} B=0, \quad s_{d}(\partial \cdot A)=0, \quad s_{d} \bar{C}=0, \quad s_{d} C=-i \mathcal{B}, \\
& s_{d} \mathcal{B}=0, \quad s_{d} \psi=-i e \bar{C} \gamma_{5} \psi, \quad s_{d} \bar{\psi}=+i e \bar{\psi} \bar{C} \gamma_{5}, \quad s_{d} E=\square \bar{C}, \\
& s_{a d} A_{\mu}=-\varepsilon_{\mu \nu} \partial^{\nu} C, \quad s_{a d} B=0, \quad s_{a d}(\partial \cdot A)=0, \quad s_{a d} C=0, \quad s_{a d} \bar{C}=+i \mathcal{B}, \\
& s_{a d} \mathcal{B}=0, \quad s_{a d} \psi=-i e C \gamma_{5} \psi, \quad s_{a d} \bar{\psi}=+i e \bar{\psi} C \gamma_{5} \quad s_{a d} E=\square C .
\end{aligned}
$$

The noteworthy points, at this stage, are (i) under the (anti-)BRST and (anti-)co-BRST transformations, it is the kinetic energy term (more precisely $E$ itself) and the gauge-fixing term (more accurately $(\partial \cdot A)$ itself) that remain invariant, respectively. (ii) The electric field $E$ and $(\partial \cdot A)$ owe their origin to the operation of cohomological operators $d$ and $\delta$ on the one-form $A=d x^{\mu} A_{\mu}$, respectively. (iii) For the (anti-)co-BRST transformations to be the symmetry transformations for (2.2), there exists the restriction that $m=0$ for the Dirac fields. There is no such restriction for the validity of the (anti-)BRST symmetry transformations. (iv) The anticommutator $\left(s_{w}=\left\{s_{b}, s_{d}\right\}=\left\{s_{a b}, s_{a d}\right\}\right)$ of the above nilpotent symmetries is a bosonic symmetry transformation $s_{w}$ (with $s_{w}^{2} \neq 0$ ) respected by the

** We follow here the notations and conventions adopted in [46]. In fact, in its full glory, a nilpotent $\left(\delta_{B}^{2}=0\right)$ BRST transformation $\delta_{B}$ is equivalent to the product of an anticommuting $(\eta C=-C \eta, \eta \bar{C}=$ $-\bar{C} \eta, \eta \psi=-\psi \eta, \eta \bar{\psi}=-\bar{\psi} \eta$ etc.) spacetime independent parameter $\eta$ and $s_{b}$ (i.e. $\delta_{B}=\eta s_{b}$ ) where $s_{b}^{2}=0$. 
Lagrangian density (2.2). These symmetry transformations are

$$
\begin{array}{ll}
s_{w} A_{\mu} & =\left(\partial_{\mu} \mathcal{B}+\varepsilon_{\mu \nu} \partial^{\nu} B\right), \quad s_{w} B=0, \quad s_{w} C=0, \\
s_{w}(\partial \cdot A) & =\square \mathcal{B}, \quad s_{w} C=0, \quad s_{w} \mathcal{B}=0, \quad s_{w} E=-\square B, \\
s_{w} \psi & =i e\left(\gamma_{5} B-\mathcal{B}\right) \psi, \quad s_{w} \bar{\psi}=+i e \bar{\psi}\left(\gamma_{5} B+\mathcal{B}\right) .
\end{array}
$$

(v) The operator algebra among the above transformations is exactly identical to the algebra obeyed by the de Rham cohomological operators (see, e.g., [36] for details). (vi) There is a ghost (scale) symmetry transformation under which only the (anti-)ghost fields transform by a scale factor (i.e. $C \rightarrow e^{-\Lambda} C, \bar{C} \rightarrow e^{\Lambda} \bar{C}$ ) and the rest of the fields do not transform at all. The infinitesimal version $s_{g}$ of this symmetry transformation is

$$
s_{g} A_{\mu}=0, \quad s_{g} B=s_{g} \mathcal{B}=0, \quad s_{g} \bar{C}=+\Lambda \bar{C}, \quad s_{g} C=-\Lambda C, \quad s_{g} \psi=s_{g} \bar{\psi}=0,
$$

where $\Lambda$ is a global parameter. (vii) The on-shell nilpotent version of the transformations in (2.3) and (2.4) do exist. For this purpose, one has to get rid of the auxiliary fields $B$ and $\mathcal{B}$ of the theory by exploiting the equations of motion (i.e. $B+(\partial \cdot A)=0, \mathcal{B}-E=0$ ). These on-shell nilpotent $\left(\tilde{s}_{(a) b}^{2}=0, \tilde{s}_{(a) d}^{2}=0\right)$ transformations are

$$
\begin{aligned}
& \tilde{s}_{b} A_{\mu}=\partial_{\mu} C, \quad \tilde{s}_{b} C=0, \quad \tilde{s}_{b} \bar{C}=-i(\partial \cdot A), \quad \tilde{s}_{b} E=0, \\
& \tilde{s}_{b} \psi=-i e C \psi, \quad \tilde{s}_{b} \bar{\psi}=-i e \bar{\psi} C, \quad \tilde{s}_{b}(\partial \cdot A)=\square C, \\
& \tilde{s}_{a b} A_{\mu}=\partial_{\mu} \bar{C}, \quad \tilde{s}_{a b} \bar{C}=0, \quad \tilde{s}_{a b} C=+i(\partial \cdot A), \quad \tilde{s}_{a b} E=0, \\
& \tilde{s}_{a b} \psi=-i e \bar{C} \psi, \quad \tilde{s}_{a b} \bar{\psi}=-i e \bar{\psi} \bar{C}, \quad \tilde{s}_{a b}(\partial \cdot A)=\square \bar{C}, \\
& \tilde{s}_{d} A_{\mu}=-\varepsilon_{\mu \nu} \partial^{\nu} \bar{C}, \quad \tilde{s}_{d} \bar{C}=0, \quad \tilde{s}_{d} C=-i E, \quad \tilde{s}_{d}(\partial \cdot A)=0, \\
& \tilde{s}_{d} \psi=-i e \bar{C} \gamma_{5} \psi, \quad \tilde{s}_{d} \bar{\psi}=+i e \bar{\psi} \bar{C} \gamma_{5}, \quad \tilde{s}_{d} E=\square \bar{C}, \\
& \tilde{s}_{a d} A_{\mu}=-\varepsilon_{\mu \nu} \partial^{\nu} C, \quad \tilde{s}_{a d} C=0, \quad \tilde{s}_{a d} \bar{C}=+i E, \quad \tilde{s}_{a d}(\partial \cdot A)=0, \\
& s_{a d} \psi=-i e C \gamma_{5} \psi, \quad \tilde{s}_{a d} \bar{\psi}=+i e \bar{\psi} C \gamma_{5}, \quad \tilde{s}_{a d} E=\square C .
\end{aligned}
$$

(vii) The above symmetry transformations are respected by the Lagrangian density $\mathcal{L}_{0}$ which can be obtained from $(2.2)$ by the substitution $B=-(\partial \cdot A), \mathcal{B}=E$, namely;

$$
\mathcal{L}_{0}=\frac{1}{2} E^{2}+\bar{\psi}\left(i \gamma^{\mu} D_{\mu}-m\right) \psi-\frac{1}{2}(\partial \cdot A)^{2}-i \partial_{\mu} \bar{C} \partial^{\mu} C
$$

(viii) It will be noted that, under the on-shell nilpotent symmetry transformations, it is only the transformations on the (anti-)ghost fields that are really affected. The transformations on the gauge- and matter fields remain intact. (ix) The above symmetry transformations are generated by the local and conserved off-shell as well as on-shell nilpotent charges $Q_{r}$ and $\tilde{Q}_{r}$, respectively. This statement can be succinctly expressed in the mathematical form as

$$
s_{r} \Omega(x)=-i\left[\Omega(x), Q_{r}\right]_{ \pm}, \quad \tilde{s}_{r} \tilde{\Omega}(x)=-i\left[\tilde{\Omega}(x), \tilde{Q}_{r}\right]_{ \pm}, \quad r=b, a b, d, a d, w, g
$$

where the local generic field $\Omega=A_{\mu}, C, \bar{C}, \psi, \bar{\psi}, B, \mathcal{B}$ and the $(+)-$ signs, as the subscripts on the (anti-)commutator $[,]_{ \pm}$, stand for $\Omega$ being (fermionic)bosonic in nature. For the on-shell nilpotent symmetry transformations in (2.7) and (2.8): $\tilde{\Omega}=A_{\mu}, C, \bar{C}, \psi, \bar{\psi}$. 
It is worthwhile to mention a set of specific discrete symmetry transformations in this interacting gauge theory which does correspond to the Hodge duality $(*)$ operation of the differential geometry. For the off-shell nilpotent (anti-)BRST invariant Lagrangian density (2.2), it can be seen that the following discrete transformations [36]

$$
\begin{gathered}
C \rightarrow \pm i \gamma_{5} \bar{C}, \quad \psi \rightarrow \psi, \quad \bar{\psi} \rightarrow \bar{\psi}, \quad A_{0} \rightarrow \pm i \gamma_{5} A_{1}, \quad A_{1} \rightarrow \pm i \gamma_{5} A_{0}, \\
\bar{C} \rightarrow \pm i \gamma_{5} C, \quad e \rightarrow \mp i e, \quad \mathcal{B} \rightarrow \mp i \gamma_{5} B, \quad B \rightarrow \mp i \gamma_{5} \mathcal{B},
\end{gathered}
$$

correspond to a symmetry transformation as the above transformations leave the Lagrangian density (2.2) invariant ${ }^{\dagger \dagger}$. It can be readily checked that the dual(co-)BRST transformations in (2.4) can be derived from their counterpart BRST transformations in (2.3) by exploiting the symmetry transformations listed in (2.11). This claim holds good for the anti-BRST and anti-co-BRST transformations as well. It is gratifying to note that, for the generic field $\Omega$, the following relationship

$$
s_{(a) d} \Omega= \pm * s_{(a) b} * \Omega
$$

is sacrosanct. Here the generic field $\Omega=A_{0}, A_{1}, B, \mathcal{B}, C, \bar{C}, \psi, \bar{\psi}$ stands for the basic fields of the Lagrangian density $(2.2)$ and the $*$ operation corresponds to the transformations (2.11). The $(+)$ - signs in the above are dictated by a couple of successive transformations (2.11) applied on $\Omega$, as is required by a consistent duality invariant theory (see, e.g., [47])

$$
*(* \Omega)= \pm \Omega \text {. }
$$

It can be readily checked that $(+)$ sign in the above corresponds to $\Omega$ being $\psi$ and $\bar{\psi}$ and $(-)$ sign stands for the rest of the basic fields of the theory. Thus, we note that the relationship between the operations $s_{d}$ and $s_{b}$ on the generic field $\Omega$ is exactly same as the relationship between the co-exterior derivative $\delta$ and the exterior derivative $d$ acting on a given differential form. It is worthwhile to emphasize that the conserved charges $Q_{(a) b}, Q_{(a) d}, W, Q_{g}$, that generate the above continuous symmetry transformations, undergo the following change under (2.11) (see, e.g., [36] for details)

$$
Q_{(a) b} \rightarrow Q_{(a) d}, \quad Q_{g} \rightarrow Q_{g}, \quad Q_{(a) d} \rightarrow Q_{(a) b}, \quad W \rightarrow W
$$

This shows that all the algebraic relations among these charges remain unchanged under (2.11). The above transformations in (2.14) should be contrasted with the corresponding transformations that exist for BRST invariant four $(3+1)$-dimensional 2-form free Abelian gauge theory $[43,44]$ where it has been shown that $Q_{(a) b} \rightarrow Q_{(a) d}, Q_{(a) d} \rightarrow-Q_{(a) b}, W \rightarrow$ $-W, Q_{g} \rightarrow-Q_{g}$ under the analogue of the discrete transformations corresponding to the Hodge duality $(*)$ operation of differential geometry. This difference in 2D and $4 \mathrm{D}$ are

\footnotetext{
${ }^{\dagger \dagger}$ In the matrix notations, the discrete transformations $A_{0} \rightarrow \pm i \gamma_{5} A_{1}$, etc., correspond to $A_{0} \rightarrow \pm i A_{1}$ and/or $A_{0} \mp i A_{1}$. Except for the exchange of signs, these transformations are identical. In the rest of the transformations (2.11), $\gamma_{5}$ has been taken into account appropriately to take care of all these sign flips.
} 
consistent with the key notions connected with the basic idea of the duality (see, e.g., [47]).

\section{Symmetries for Gauge- and (Anti-)ghost Fields: Usual Superfield Formalism}

We begin here with a four $(2+2)$-dimensional supermanifold parametrized by the superspace coordinates $Z^{M}=\left(x^{\mu}, \theta, \bar{\theta}\right)$ where $x^{\mu}(\mu=0,1)$ are a couple of even (bosonic) spacetime coordinates and $\theta$ and $\bar{\theta}$ are the two odd (Grassmannian) coordinates (with $\theta^{2}=\bar{\theta}^{2}=$ $0, \theta \bar{\theta}+\bar{\theta} \theta=0)$. On this supermanifold, one can define a super 1 -form $\tilde{A}=d Z^{M} \tilde{A}_{M}$ with the supervector superfield $\tilde{A}_{M}$ (i.e. $\tilde{A}_{M}=\left(B_{\mu}(x, \theta, \bar{\theta}), \Phi(x, \theta, \bar{\theta}), \bar{\Phi}(x, \theta, \bar{\theta})\right)$. Here $B_{\mu}, \Phi, \bar{\Phi}$ are the component multiplet superfields of the supervector superfield $\tilde{A}_{M}$ [14]. The general superfields $\left(B_{\mu}, \Phi, \bar{\Phi}\right)(x, \theta, \bar{\theta})$ can be expanded in terms of the basic fields $\left(A_{\mu}, C, \bar{C}\right)(x)$ and auxiliary fields $(B, \mathcal{B})(x)$ of $(2.2)$ and some extra secondary fields as

$$
\begin{aligned}
& B_{\mu}(x, \theta, \bar{\theta})=A_{\mu}(x)+\theta \bar{R}_{\mu}(x)+\bar{\theta} R_{\mu}(x)+i \theta \bar{\theta} S_{\mu}(x), \\
& \Phi(x, \theta, \bar{\theta})=C(x)+i \theta \bar{B}(x)-i \bar{\theta} \mathcal{B}(x)+i \theta \bar{\theta} s(x), \\
& \bar{\Phi}(x, \theta, \bar{\theta})=\bar{C}(x)-i \theta \overline{\mathcal{B}}(x)+i \bar{\theta} B(x)+i \theta \bar{\theta} \bar{s}(x)
\end{aligned}
$$

It is straightforward to note that the local fields $R_{\mu}(x), \bar{R}_{\mu}(x), C(x), \bar{C}(x), s(x), \bar{s}(x)$ are the fermionic (anti-commuting) in nature and the bosonic (commuting) local fields in (3.1) are: $A_{\mu}(x), S_{\mu}(x), \mathcal{B}(x), \overline{\mathcal{B}}(x), B(x), \bar{B}(x)$. It is obvious that, in the above expansion, the bosonic- and fermionic degrees of freedom do match. This requirement is essential for the sanctity of any arbitrary supersymmetric field theory in the superfield formulation. In fact, all the secondary fields will be expressed in terms of basic fields due to the restrictions emerging from the application of horizontality condition (i.e. $\tilde{F}=F$ ), namely;

$$
\tilde{F}=\frac{1}{2}\left(d Z^{M} \wedge d Z^{N}\right) \tilde{F}_{M N} \equiv \tilde{d} \tilde{A}=d A \equiv \frac{1}{2}\left(d x^{\mu} \wedge d x^{\nu}\right) F_{\mu \nu}=F,
$$

where the super exterior derivative $\tilde{d}$ and the connection super one-form $\tilde{A}$ are defined as

$$
\begin{aligned}
& \tilde{d}=d Z^{M} \partial_{M}=d x^{\mu} \partial_{\mu}+d \theta \partial_{\theta}+d \bar{\theta} \partial_{\bar{\theta}}, \\
& \tilde{A}=d Z^{M} \tilde{A}_{M}=d x^{\mu} B_{\mu}(x, \theta, \bar{\theta})+d \theta \bar{\Phi}(x, \theta, \bar{\theta})+d \bar{\theta} \Phi(x, \theta, \bar{\theta}) .
\end{aligned}
$$

In physical language, the requirement (3.2) implies that the gauge invariant physical field $E$, derived from the curvature term $F_{\mu \nu}$, does not get any contribution from the Grassmannian variables. In other words, the physical electric field $E$ for $2 \mathrm{D}$ QED remains intact in the superfield formulation. Mathematically, the condition (3.2) implies the "flatness" of all

the components of the super curvature (2-form) tensor $\tilde{F}_{M N}$ that are directed along the $\theta$ and/or $\bar{\theta}$ directions of the supermanifold. To clearly see it, first we expand $\tilde{d} \tilde{A}$ as

$$
\begin{aligned}
\tilde{d} \tilde{A} & =\left(d x^{\mu} \wedge d x^{\nu}\right)\left(\partial_{\mu} B_{\nu}\right)-(d \theta \wedge d \theta)\left(\partial_{\theta} \bar{\Phi}\right)+\left(d x^{\mu} \wedge d \bar{\theta}\right)\left(\partial_{\mu} \Phi-\partial_{\bar{\theta}} B_{\mu}\right) \\
& -(d \theta \wedge d \bar{\theta})\left(\partial_{\theta} \Phi+\partial_{\bar{\theta}} \bar{\Phi}\right)+\left(d x^{\mu} \wedge d \theta\right)\left(\partial_{\mu} \bar{\Phi}-\partial_{\theta} B_{\mu}\right)-(d \bar{\theta} \wedge d \bar{\theta})\left(\partial_{\bar{\theta}} \Phi\right) .
\end{aligned}
$$

Ultimately, the application of soul-flatness (horizontality) condition $(\tilde{d} \tilde{A}=d A)$ yields $[27]$

$$
\begin{aligned}
& R_{\mu}(x)=\partial_{\mu} C(x), \quad \bar{R}_{\mu}(x)=\partial_{\mu} \bar{C}(x), \quad s(x)=\bar{s}(x)=0, \\
& S_{\mu}(x)=\partial_{\mu} B(x) \quad B(x)+\bar{B}(x)=0, \quad \mathcal{B}(x)=\overline{\mathcal{B}}(x)=0 .
\end{aligned}
$$


The insertion of all the above values in the expansion (3.1) leads to the derivation of the (anti-)BRST symmetries for the gauge- and (anti-)ghost fields of the Abelian gauge theory. In addition, this exercise provides the physical interpretation for the (anti-)BRST charges $Q_{(a) b}$ as the generators (cf. (2.10)) of translations (i.e. $\operatorname{Lim}_{\bar{\theta} \rightarrow 0}(\partial / \partial \theta), \operatorname{Lim}_{\theta \rightarrow 0}(\partial / \partial \bar{\theta})$ ) along the Grassmannian directions of the supermanifold. Both these observations can be succinctly expressed, in a combined way, by re-writing the super expansion (3.1) as

$$
\begin{aligned}
B_{\mu}(x, \theta, \bar{\theta}) & =A_{\mu}(x)+\theta\left(s_{a b} A_{\mu}(x)\right)+\bar{\theta}\left(s_{b} A_{\mu}(x)\right)+\theta \bar{\theta}\left(s_{b} s_{a b} A_{\mu}(x)\right) \\
\Phi(x, \theta, \bar{\theta}) & =C(x)+\theta\left(s_{a b} C(x)\right)+\bar{\theta}\left(s_{b} C(x)\right)+\theta \bar{\theta}\left(s_{b} s_{a b} C(x)\right) \\
\bar{\Phi}(x, \theta, \bar{\theta}) & =\bar{C}(x)+\theta\left(s_{a b} \bar{C}(x)\right)+\bar{\theta}\left(s_{b} \bar{C}(x)\right)+\theta \bar{\theta}\left(s_{b} s_{a b} \bar{C}(x)\right) .
\end{aligned}
$$

The on-shell nilpotent (anti-)BRST symmetry transformations (2.7) can also be derived in the framework of the usual superfield formulation [27]. For this purpose, we take the chiral limit (i.e. $\theta \rightarrow 0$ ) of the general expansion in (3.1) and the general definitions in (3.3), as

$$
\begin{aligned}
& B_{\mu}^{(c)}(x, \bar{\theta})=A_{\mu}(x)+\bar{\theta} R_{\mu}(x), \quad \Phi^{(c)}(x, \bar{\theta})=C(x)-i \bar{\theta} \mathcal{B}(x), \\
& \bar{\Phi}^{(c)}(x, \bar{\theta})=\bar{C}(x)+i \bar{\theta} B(x), \quad \tilde{d}_{(c)}=d x^{\mu} \partial_{\mu}+d \bar{\theta} \partial_{\bar{\theta}}, \\
& \left.\tilde{A}\right|_{(c)}=d x^{\mu} B_{\mu}^{(c)}(x, \bar{\theta})+d \bar{\theta} \Phi^{(c)}(x, \bar{\theta}) .
\end{aligned}
$$

It can be noted that the basic tenets of supersymmetry are satisfied here too. The bosonic $\left(A_{\mu}, B, \mathcal{B}\right)$ and fermionic $\left(R_{\mu}, C, \bar{C}\right)$ degrees of freedom do match on the chiral three $(2+1)$ dimensional super sub-manifold parametrized by two even $\left(x_{0}, x_{1}\right)$ variables and one odd $\bar{\theta}$ variable. The explicit form of the super curvature 2 -form on the above super sub-manifold, constructed by $\left.\tilde{d}\right|_{(c)}$ and $\left.\tilde{A}\right|_{(c)}$, is

$$
\begin{aligned}
\left.\left.\tilde{d}\right|_{(c)} \tilde{A}\right|_{(c)} & =\left(d x^{\mu} \wedge d x^{\nu}\right)\left(\partial_{\mu} B_{\nu}^{(c)}\right)-(d \bar{\theta} \wedge d \bar{\theta})\left(\partial_{\bar{\theta}} \Phi^{(c)}\right) \\
& +\left(d x^{\mu} \wedge d \bar{\theta}\right)\left(\partial_{\mu} \Phi^{(c)}-\partial_{\bar{\theta}} B_{\mu}^{(c)}\right) .
\end{aligned}
$$

The horizontality condition $\left.\left.\tilde{d}\right|_{(c)} A\right|_{(c)}=d A$ leads to the following results:

$$
R_{\mu}(x)=\partial_{\mu} C(x), \quad \mathcal{B}(x)=0 .
$$

It is evident that the above horizontality condition does not fix the value of the auxiliary field $B$ in terms of the basic fields of the Lagrangian density (2.9). However, the equation of motion $B+(\partial \cdot A)=0$, emerging from the Lagrangian density (2.2), comes to our rescue. The insertions of the above values in the expansion (3.7) lead to the following

$$
\begin{aligned}
& B_{\mu}^{(c)}(x, \bar{\theta})=A_{\mu}(x)+\bar{\theta}\left(\tilde{s}_{b} A_{\mu}(x)\right), \quad \Phi^{(c)}(x, \bar{\theta})=C(x)+\bar{\theta}\left(\tilde{s}_{b} C(x)\right), \\
& \bar{\Phi}^{(c)}(x, \bar{\theta})=\bar{C}(x)+\bar{\theta}\left(\tilde{s}_{b} \bar{C}(x)\right) .
\end{aligned}
$$

Comparison with (2.10) leads to the geometrical interpretation for the on-shell nilpotent BRST charge $\tilde{Q}_{b}$ as the translational generator $(\partial / \partial \bar{\theta})$, for the above chiral superfields, along the $\bar{\theta}$-direction of the $(2+1)$-dimensional chiral super sub-manifold. The process of translation of the chiral superfields produces the on-shell nilpotent transformations $\tilde{s}_{b}$ for the usual fields $A_{\mu}(x), C(x), \bar{C}(x)$ defined on the ordinary manifold. Now, we discuss 
the derivation of the on-shell nilpotent anti-BRST symmetry transformations $\tilde{s}_{a b}$. For this purpose, we take the anti-chiral (i.e $\bar{\theta} \rightarrow 0$ ) limit of the general expansion (3.1) as well the general definitions (3.3), as

$$
\begin{aligned}
& B_{\mu}^{(a c)}(x, \theta)=A_{\mu}(x)+\theta \bar{R}_{\mu}(x), \quad \Phi^{(a c)}(x, \theta)=C(x)-i \theta B(x), \\
& \bar{\Phi}^{(a c)}(x, \theta)=\bar{C}(x)-i \theta \overline{\mathcal{B}}(x), \quad \tilde{d}_{(a c)}=d x^{\mu} \partial_{\mu}+d \theta \partial_{\theta}, \\
& \left.\tilde{A}\right|_{(a c)}=d x^{\mu} B_{\mu}^{(a c)}(x, \theta)+d \theta \bar{\Phi}^{(a c)}(x, \theta) .
\end{aligned}
$$

In the above expansion, it should be noted that (i) the fermionic $\left(\bar{R}_{\mu}, C, \bar{C}\right)$ and bosonic $\left(A_{\mu}, B, \overline{\mathcal{B}}\right)$ degrees of freedom do match, and (ii) we have taken into account $B(x)+\bar{B}(x)=$ 0 , from our earlier experience in (3.5), for the expansion of $\Phi^{(a c)}$. One can construct the super 2-form curvature $\left.\left.\tilde{d}\right|_{(a c)} \tilde{A}\right|_{(a c)}$, from the above definitions, as follows

$$
\begin{aligned}
\left.\left.\tilde{d}\right|_{(a c)} \tilde{A}\right|_{(a c)} & =\left(d x^{\mu} \wedge d x^{\nu}\right)\left(\partial_{\mu} B_{\nu}^{(a c)}\right)-(d \theta \wedge d \theta)\left(\partial_{\theta} \bar{\Phi}^{(a c)}\right) \\
& +\left(d x^{\mu} \wedge d \theta\right)\left(\partial_{\mu} \bar{\Phi}^{(a c)}-\partial_{\theta} B_{\mu}^{(a c)}\right) .
\end{aligned}
$$

The imposition of the horizontality condition $\left.\left.\tilde{d}\right|_{(a c)} \tilde{A}\right|_{(a c)}=d A$ yields the following results

$$
\bar{R}_{\mu}(x)=\partial_{\mu} \bar{C}(x), \quad \overline{\mathcal{B}}(x)=0 .
$$

It is clear that the above condition (3.13) does not fix the auxiliary field $B(x)$ present in the expansion of $\Phi^{(a c)}$ in (3.11). However, the equation of motion $B+(\partial \cdot A)=0$ derived from the Lagrangian density (2.2) turns out to be helpful for our purpose. The substitutions of the results in (3.13) and $B=-(\partial \cdot A)$ in the expansion (3.11) leads to

$$
\begin{aligned}
& B_{\mu}^{(a c)}(x, \theta)=A_{\mu}(x)+\theta\left(\tilde{s}_{a b} A_{\mu}(x)\right), \quad \Phi^{(a c)}(x, \theta)=C(x)+\theta\left(\tilde{s}_{a b} C(x)\right), \\
& \bar{\Phi}^{(a c)}(x, \theta)=\bar{C}(x)+\theta\left(\tilde{s}_{a b} \bar{C}(x)\right) .
\end{aligned}
$$

The above expansion explicitly explains the geometrical origin for the on-shell nilpotent symmetry transformations $\tilde{s}_{a b}$ as the translation generator $(\partial / \partial \theta)$ along the $\theta$-direction of an anti-chiral super sub-manifold. The process of translation of the above anti-chiral superfields induces the internal on-shell nilpotent anti-BRST transformations on the fields $A_{\mu}(x), C(x), \bar{C}(x)$ defined on the ordinary manifold.

To obtain the (anti-)co-BRST transformations on the gauge- and (anti-)ghost fields, we exploit the dual-horizontality condition $\tilde{\delta} \tilde{A}=\delta A$ on the $(2+2)$-dimensional supermanifold where $\tilde{\delta}=-\star \tilde{d} \star$ is the super co-exterior derivative on the four $(2+2)$-dimensional supermanifold and $\delta=-* d *$ is the co-exterior derivative on the ordinary 2D manifold. The Hodge duality operations on the supermanifold and ordinary manifold are denoted by $\star$ and $*$, respectively. The $\star$ operations on the super differentials $\left(d Z^{M}\right)$ and their wedge products $\left(d Z^{M} \wedge d Z^{N}\right)$, etc., defined on the $(2+2)$-dimensional supermanifold, are [48]

$$
\begin{array}{ll}
\star\left(d x^{\mu}\right)=\varepsilon^{\mu \nu}\left(d x_{\nu} \wedge d \theta \wedge d \bar{\theta}\right), & \star(d \theta)=\frac{1}{2 !} \varepsilon^{\mu \nu}\left(d x_{\mu} \wedge d x_{\nu} \wedge d \bar{\theta}\right), \\
\star(d \bar{\theta})=\frac{1}{2 !} \varepsilon^{\mu \nu}\left(d x_{\mu} \wedge d x_{\nu} \wedge d \theta\right), & \star\left(d x^{\mu} \wedge d x^{\nu}\right)=\varepsilon^{\mu \nu}(d \theta \wedge d \bar{\theta}), \\
\star\left(d x^{\mu} \wedge d \theta\right)=\varepsilon^{\mu \nu}\left(d x_{\nu} \wedge d \bar{\theta}\right), & \star\left(d x^{\mu} \wedge d \bar{\theta}\right)=\varepsilon^{\mu \nu}\left(d x_{\nu} \wedge d \theta\right), \\
\star(d \theta \wedge d \theta)=\frac{1}{2 !} s^{\theta \theta} \varepsilon^{\mu \nu}\left(d x_{\mu} \wedge d x_{\nu}\right), & \star(d \theta \wedge d \bar{\theta})=\frac{1}{2 !} \varepsilon^{\mu \nu}\left(d x_{\mu} \wedge d x_{\nu}\right), \\
\star(d \bar{\theta} \wedge d \bar{\theta})=\frac{1}{2 !} s^{\theta \bar{\theta}} \varepsilon^{\mu \nu}\left(d x_{\mu} \wedge d x_{\nu}\right), & \star\left(d x_{\mu} \wedge d \theta \wedge d \bar{\theta}\right)=\varepsilon_{\mu \nu}\left(d x^{\nu}\right), \\
\star\left(d x_{\mu} \wedge d x_{\nu} \wedge d \theta \wedge d \bar{\theta}\right)=\varepsilon_{\mu \nu}, & \star\left(d x_{\mu} \wedge d x_{\nu} \wedge d \theta\right)=\varepsilon_{\mu \nu}(d \bar{\theta}), \\
\star\left(d x_{\mu} \wedge d x_{\nu} \wedge d \bar{\theta}\right)=\varepsilon_{\mu \nu}(d \theta), & \star\left(d x_{\mu} \wedge d x_{\nu} \wedge d \theta \wedge d \theta\right)=\varepsilon_{\mu \nu} s^{\theta \theta},
\end{array}
$$


where s's are the symmetric constant quantities on the Grassmannian submanifold of the four $(2+2)$-dimensional supermanifold. They are introduced to take care of the fact that two successive $\star$ operation on any differential (or its wedge products) should yield the same differential (or its wedge products) (see, e.g., [48] for details). In the above we have collected only a few of the $\star$ operations. The other appropriate $\star$ operations can be computed in an analogous manner. With the above inputs, it can be checked that the superscalar superfield $\tilde{\delta} \tilde{A}=-\star \tilde{d} \star \tilde{A}$, expressed in an explicit form, turns out to be

$$
\tilde{\delta} \tilde{A}=(\partial \cdot B)+\left(\partial_{\theta} \bar{\Phi}+\partial_{\bar{\theta}} \Phi\right)+s^{\theta \theta}\left(\partial_{\theta} \Phi\right)+s^{\bar{\theta} \bar{\theta}}\left(\partial_{\bar{\theta}} \bar{\Phi}\right) .
$$

It is to be noted that in the above computation of $\tilde{d} \star \tilde{A}$, we have dropped all the terms that contain (i) more than two differentials of spacetime in the wedge products, and (ii) more than two differentials of Grassmann variables in the wedge products. Having done that, we have applied another $\star$ operation on it (i.e. $-\star \tilde{d} \star \tilde{A}=\tilde{\delta} \tilde{A}$ ). Ultimately, the dual-horizontality restriction $\tilde{\delta} \tilde{A}=\delta A$ produces the following restrictions

$$
\partial_{\theta} \Phi=0, \quad \partial_{\bar{\theta}} \bar{\Phi}=0, \quad(\partial \cdot B)+\partial_{\theta} \bar{\Phi}+\partial_{\bar{\theta}} \Phi=(\partial \cdot A),
$$

where, as is evident, the r.h.s. of the last entry in the above equation is due to $\delta A=(\partial \cdot A)$. Exploiting the super expansions of (3.1), we obtain

$$
\begin{aligned}
& (\partial \cdot R)(x)=(\partial \cdot \bar{R})(x)=(\partial \cdot S)(x)=0, \quad s(x)=\bar{s}(x)=0, \\
& B(x)=0, \quad \bar{B}(x)=0, \quad \mathcal{B}(x)+\overline{\mathcal{B}}(x)=0 .
\end{aligned}
$$

It is clear from the above that we cannot get a unique solution for $R_{\mu}, \bar{R}_{\mu}$ and $S_{\mu}$ in terms of the basic fields of the Lagrangian density (2.2). This is why there are non-local and non-covariant solutions for these in the case of QED in 4D. A detailed discussion on this issue can be found in our recent work [48]. It is interesting, however, to point out that for 2D QED, we have a local and covariant solution for the above restrictions, as

$$
R_{\mu}=-\varepsilon_{\mu \nu} \partial^{\nu} \bar{C}, \quad \bar{R}_{\mu}=-\varepsilon_{\mu \nu} \partial^{\nu} C, \quad S_{\mu}=+\varepsilon_{\mu \nu} \partial^{\nu} \mathcal{B} .
$$

With the above insertions, it can be easily checked that the expansion (3.1) becomes

$$
\begin{aligned}
& B_{\mu}(x, \theta, \bar{\theta})=A_{\mu}(x)+\theta\left(s_{a d} A_{\mu}(x)\right)+\bar{\theta}\left(s_{d} A_{\mu}(x)\right)+\theta \bar{\theta}\left(s_{d} s_{a d} A_{\mu}(x)\right), \\
& \Phi(x, \theta, \bar{\theta})=C(x)+\theta\left(s_{a d} C(x)\right)+\bar{\theta}\left(s_{d} C(x)\right)+\theta \bar{\theta}\left(s_{d} s_{a d} C(x)\right), \\
& \bar{\Phi}(x, \theta, \bar{\theta})=\bar{C}(x)+\theta\left(s_{a d} \bar{C}(x)\right)+\bar{\theta}\left(s_{d} \bar{C}(x)\right)+\theta \bar{\theta}\left(s_{d} s_{a d} \bar{C}(x)\right) .
\end{aligned}
$$

Thus, the geometrical interpretation for the generators $Q_{(a) d}$ of the (anti-)co-BRST symmetries is identical to that of the (anti-)BRST charges $Q_{(a) b}$. However, there is a clear-cut distinction between $Q_{(a) d}$ and $Q_{(a) b}$ when the transformations on the (anti-)ghost fields are considered. For instance, the BRST charge $Q_{b}$ generates a symmetry transformation such that the superfield $\bar{\Phi}(x, \theta, \bar{\theta})$ becomes anti-chiral and the superfield $\Phi(x, \theta, \bar{\theta})$ becomes an ordinary local field $C(x)$. In contrast, the co-BRST charge $Q_{d}$ generates a symmetry transformation under which just the opposite of the above happens. Similarly, the distinction between $Q_{a b}$ and $Q_{a d}$ can be argued where one of the above superfields becomes chiral. 
Let us dwell a bit on the derivations of the on-shell nilpotent (anti-)co-BRST symmetry transformations $\tilde{s}_{(a) d}$, listed in (2.8), by exploiting the superfield formulation. For this purpose, we focus on the expansions and definitions, listed in (3.7), for the derivation of the BRST transformations $\tilde{s}_{b}$. With these inputs from (3.7), one can compute the following

$$
\left.\left.\tilde{\delta}\right|_{(c)} \tilde{A}\right|_{(c)}=\left(\partial \cdot B^{(c)}\right)+s^{\bar{\theta} \bar{\theta}}\left(\partial_{\bar{\theta}} \bar{\Phi}^{(c)}\right),
$$

where $\left.\tilde{\delta}\right|_{(c)}=-\left.\star \tilde{d}\right|_{(c)} \mid \star$. It will be noted that the expression in (3.21) is the chiral limit $(\theta \rightarrow 0)$ of the most general expression in (3.16). The dual-horizontality condition $\left.\left.\tilde{\delta}\right|_{(c)} \tilde{A}\right|_{(c)}=\delta A$ on the three $(2+1)$-dimensional chiral super sub-manifold leads to the following conditions on the chiral superfields

$$
\partial_{\bar{\theta}} \bar{\Phi}^{(c)}=0, \quad\left(\partial \cdot B^{(c)}\right)=(\partial \cdot A),
$$

which imply a couple of restrictions on the component fields as listed below

$$
(\partial \cdot R)(x)=0, \quad B(x)=0 .
$$

It is evident from the above that there is no unique solution to the condition $(\partial \cdot R)=0$. One can have a non-local and non-covariant solution to the above as has been derived for the QED in any arbitrary dimensions [37,38]. However, it is obvious that for the 2D QED, there exists a local and covariant solution because $R_{\mu}$ can be chosen to be: $R_{\mu}=-\varepsilon_{\mu \nu} \partial^{\nu} \bar{C}$. It should be noted here that the auxiliary field $\mathcal{B}(x)$ is not fixed by the above dual-horizontality condition, in terms of the basic fields of the Lagrangian density (2.1). The equation of motion $E-\mathcal{B}=0$ from the Lagrangian density (2.2), however, turns out to be useful for our purpose. The insertions of the above values in the expansion (3.7) leads to

$$
\begin{aligned}
& B_{\mu}^{(c)}(x, \bar{\theta})=A_{\mu}(x)+\bar{\theta}\left(\tilde{s}_{d} A_{\mu}(x)\right), \quad \Phi^{(c)}(x, \bar{\theta})=C(x)+\bar{\theta}\left(\tilde{s}_{d} C(x)\right), \\
& \bar{\Phi}^{(c)}(x, \bar{\theta})=\bar{C}(x)+\bar{\theta}\left(\tilde{s}_{d} \bar{C}(x)\right) .
\end{aligned}
$$

To derive the on-shell nilpotent anti-BRST symmetry transformations of (2.8), we take the anti-chiral limit of the most general expansion (3.1) and most general definitions in (3.3). These are listed in (3.11). With these inputs from (3.11), we can define the analogue of (3.21) in terms of the anti-chiral super co-exterior derivative $\left.\tilde{\delta}\right|_{(a c)}$ and connection $\left.\tilde{A}\right|_{(a c)}$, as

$$
\left.\left.\tilde{\delta}\right|_{(a c)} \tilde{A}\right|_{(a c)}=\left(\partial \cdot B^{(a c)}\right)+s^{\theta \theta}\left(\partial_{\theta} \Phi^{(a c)}\right),
$$

where $\tilde{\delta}_{(a c)}=-\left.\star \tilde{d}\right|_{(a c)} \star$. The dual-horizontality condition $\left.\left.\tilde{\delta}\right|_{(a c)} \tilde{A}\right|_{(a c)}=\delta A$ implies

$$
\partial_{\theta} \Phi^{(a c)}=0 \Rightarrow B(x)=0, \quad\left(\partial \cdot B^{(a c)}\right)=(\partial \cdot A) \Rightarrow(\partial \cdot \bar{R})=0 .
$$

At this juncture, there are two comments in order. First, the local and covariant solution for the restriction $(\partial \cdot \bar{R})=0$ exists if we take into account $\bar{R}_{\mu}(x)=-\varepsilon_{\mu \nu} \partial^{\nu} C$. Second, the above dual-horizontality condition does not fix the value of the auxiliary field $\mathcal{B}(x)$. 
However, from the equation of motion from the Lagrangian density (2.2), it is clear that $\mathcal{B}=E$. The substitution of these values (i.e. $B=0, \mathcal{B}=E, \bar{R}_{\mu}=-\varepsilon_{\mu \nu} \partial^{\nu} C$ ) leads to the following expansion for the anti-chiral superfields of (3.11):

$$
\begin{aligned}
& B_{\mu}^{(a c)}(x, \theta)=A_{\mu}(x)+\theta\left(\tilde{s}_{a d} A_{\mu}(x)\right), \quad \Phi^{(a c)}(x, \theta)=C(x)+\bar{\theta}\left(\tilde{s}_{a d} C(x)\right), \\
& \bar{\Phi}^{(c)}(x, \theta)=\bar{C}(x)+\bar{\theta}\left(\tilde{s}_{a d} \bar{C}(x)\right) .
\end{aligned}
$$

The expansions in (3.27) and (3.24) provide the geometrical interpretation for the onshell nilpotent (anti-)co-BRST charges as the translation generators $((\partial / \partial \theta)(\partial / \partial \bar{\theta}))$ along the Grassmannian $(\theta) \bar{\theta}$-directions of the three $(2+1)$-dimensional (anti-)chiral super submanifolds parametrized by $x^{\mu}$ and $(\theta) \bar{\theta}$. For this interpretation, it is essential to exploit the general expression for the on-shell nilpotent symmetry transformations given in (2.10). To be more precise, the translations of the (anti-)chiral superfields along the $(\theta) \bar{\theta}$-directions of the (anti-)chiral super sub-manifold generates the internal symmetry transformations $\tilde{s}_{(a) d}$ on the usual fields $A_{\mu}(x), C(x), \bar{C}(x)$.

\section{Nilpotent Symmetries for Dirac fields: Augmented Superfield Formalism}

We have exploited the (dual-)horizontality conditions (i.e. $\tilde{\delta} \tilde{A}=\delta A, \tilde{d} \tilde{A}=d A$ ) on the most general $(2+2)$-dimensional (super)manifolds to derive the (anti-)co-BRST- and (anti-)BRST symmetry transformations for the gauge- and (anti-)ghost fields of the interacting gauge theory in the previous Section. It is evident that the horizontality- and dual-horizontality conditions (which physically owe their origin to the gauge invariance of the electric field $E$ and the dual-gauge invariance of the gauge-fixing term, respectively) do not shed any light on the nilpotent symmetry transformations that exist for the matter fields $(\psi, \bar{\psi})$ of the interacting theory. However, we do know that there exist an additional gauge invariant quantity $J_{\mu}=\bar{\psi} \gamma_{\mu} \psi$ and an additional $\gamma_{5}$-chiral (i.e. $m=0$ ) gauge invari-

ant quantity $J_{\mu}^{(5)}=\bar{\psi} \gamma_{\mu} \gamma_{5} \psi$ in the theory. These invariant quantities are constructed by the matter fields of the interacting theory (and are not explicitly written in terms of the gauge field $A_{\mu}$ and any of the de Rham cohomological operators). To establish that the invariance of these currents on the (super)manifolds, leads to the derivation of the nilpotent symmetry transformations on the matter fields, we define the superfields $(\Psi, \bar{\Psi})(x, \theta, \bar{\theta}))$ corresponding to the ordinary Dirac fields $(\psi, \bar{\psi})(x)$. These can be expanded along all the four independent directions (i.e. $\hat{\mathbf{1}}, \theta, \bar{\theta}, \theta \bar{\theta})$ of the most general $(2+2)$-dimensional supermanifold as [31-33]

$$
\begin{aligned}
& \Psi(x, \theta, \bar{\theta})=\psi(x)+i \theta \bar{b}_{1}(x)+i \bar{\theta} b_{2}(x)+i \theta \bar{\theta} f(x), \\
& \bar{\Psi}(x, \theta, \bar{\theta})=\bar{\psi}(x)+i \theta \bar{b}_{2}(x)+i \bar{\theta} b_{1}(x)+i \theta \bar{\theta} \bar{f}(x) .
\end{aligned}
$$

It is obvious that, in the limit $(\theta, \bar{\theta}) \rightarrow 0$, we get back the Dirac fields $(\psi, \bar{\psi})$ of the Lagrangian density (2.1). Furthermore, the number of bosonic fields $\left(b_{1}, \bar{b}_{1}, b_{2}, \bar{b}_{2}\right)$ match with the fermionic fields $(\psi, \bar{\psi}, f, \bar{f})$ so that the above expansion is consistent with the basic tenets of supersymmetry for the present superfield formulation to be correct. 
For the sake of one of the the simplest ways to derive the nilpotent BRST symmetry transformations $s_{b}$ for the matter fields, we take the chiral limit (i.e. $\theta \rightarrow 0$ ) of the expansion (4.1) as illustrated below

$$
\Psi^{(c)}(x, \bar{\theta})=\psi(x)+i \bar{\theta} b_{2}(x), \quad \bar{\Psi}^{(c)}(x, \bar{\theta})=\bar{\psi}(x)+i \bar{\theta} b_{1}(x)
$$

It can be readily seen that the fermionic $(\psi, \bar{\psi})$ and bosonic $\left(b_{1}, b_{2}\right)$ degrees of freedom do match on the chiral supermanifold parameterized by the bosonic variable $x^{\mu}$ and one Grassmannian variable $\bar{\theta}$. From the above chiral expansion, we can construct the chiral supercurrent $\tilde{J}_{\mu}^{(c)}(x, \bar{\theta})$ and expand it along $\bar{\theta}$-direction as

$$
\tilde{J}_{\mu}^{(c)}(x, \bar{\theta})=\bar{\Psi}^{(c)}(x, \bar{\theta}) \gamma_{\mu} \Psi^{(c)}(x, \bar{\theta})=J_{\mu}(x)+i \bar{\theta}\left(b_{1} \gamma_{\mu} \psi-\bar{\psi} \gamma_{\mu} b_{2}\right)
$$

Requirement of the invariance of the (super)currents on the (super)manifolds implies

$$
\tilde{J}_{\mu}^{(c)}(x, \bar{\theta})=J_{\mu}(x) \Rightarrow b_{1} \gamma_{\mu} \psi=\bar{\psi} \gamma_{\mu} b_{2}
$$

The above equality is satisfied if the bosonic components $b_{1}$ and $b_{2}$ are proportional to the fermionic fields $\bar{\psi}$ and $\psi$, respectively. To make the latter pair $\bar{\psi}$ and $\psi$ bosonic in nature, we have to bring in the ghost fields of the theory so that $b_{1} \sim \bar{\psi} C, b_{2} \sim C \psi$. In conformity with the transformations in $(2.3)$, we take $b_{1}=-e \bar{\psi} C, b_{2}=-e C \psi$. Inserting these values in (4.3), we obtain, vis-⿳亠丷⿵冂-vis (2.3), the following expansion

$$
\Psi^{(c)}(x, \bar{\theta})=\psi(x)+\bar{\theta}\left(s_{b} \psi(x)\right), \quad \bar{\Psi}^{(c)}(x, \bar{\theta})=\bar{\psi}(x)+\bar{\theta}\left(s_{b} \bar{\psi}(x)\right) .
$$

This clearly establishes the geometrical interpretation for the nilpotent BRST charge $Q_{b}$ (and the corresponding transformation $\left.s_{b}\right)$ as the translation generator $(\partial / \partial \bar{\theta})$ for the chiral superfields $\Psi^{(c)}(x, \bar{\theta})$ and $\bar{\Psi}^{(c)}(x, \bar{\theta})$ along the $\bar{\theta}$-direction of the $(2+1)$-dimensional chiral super sub-manifold (parametrized by $x^{\mu}$ and $\bar{\theta}$ ). It will be noted that the restriction in (4.4) is not put by hand. Rather, it is the inherent property of the theory itself which is quite natural. To justify this statement, it can be seen that (4.3) can be re-expressed as

$$
\tilde{J}_{\mu}^{(c)}(x, \bar{\theta})=\bar{\Psi}^{(c)}(x, \bar{\theta}) \gamma_{\mu} \Psi^{(c)}(x, \bar{\theta})=J_{\mu}(x)+\bar{\theta}\left(s_{b} J_{\mu}(x)\right) .
$$

However, it can be checked, using the explicit nilpotent BRST transformations in (2.3), that $s_{b} J_{\mu}(x)=0$. Thus, the restriction $\tilde{J}_{\mu}^{(c)}(x, \bar{\theta})=J_{\mu}(x)$ is not imposed by hand from outside. Rather, it is a natural restriction on the supermanifold which preserves (i) the geometrical interpretation for the $Q_{b}$ and $s_{b}$ as the translation generator $(\partial / \partial \bar{\theta})$, and (ii) the nilpotency of $s_{b}^{2}=0$ (and $Q_{b}^{2}=0$ ) as a couple of successive translations (i.e. $(\partial / \partial \bar{\theta})^{2}=0$ ) along the $\bar{\theta}$-direction of the supermanifold. This pair of geometrical properties (i) and (ii) is exactly same as in the previous Section where the nilpotent symmetries for the gauge- and (anti-)ghost fields were derived. Furthermore, it should be noted that this chiral superfield formulation does not shed any light on the anticommutativity $s_{b} s_{a b}+s_{a b} s_{b}=0$ of the (anti-)BRST transformations $s_{(a) b}$ and corresponding charges $Q_{b} Q_{a b}+Q_{a b} Q_{b}=0$. 
One of the easiest ways to derive the nilpotent $\left(s_{a b}^{2}=0\right)$ anti-BRST transformations $s_{a b}$ for the matter fields $\psi$ and $\bar{\psi}$ of the Lagrangian density (2.2) is to begin with the anti-chiral limit $(\bar{\theta} \rightarrow 0)$ of the expansion in (4.1) as listed below

$$
\Psi^{(a c)}(x, \theta)=\psi(x)+i \theta \bar{b}_{1}(x), \quad \bar{\Psi}^{(a c)}(x, \theta, \theta)=\bar{\psi}(x)+i \theta \bar{b}_{2}(x) .
$$

Here too, the bosonic degrees of freedom $\left(\bar{b}_{1}, \bar{b}_{2}\right)$ and fermionic degrees of freedom $(\psi, \bar{\psi})$ match as per the requirement of the above theory to be consistent with the basic ideas of supersymmetry. One can construct the super anti-chiral matter current

$$
\begin{aligned}
\tilde{J}_{\mu}^{(a c)}(x, \theta) & =\bar{\Psi}^{(a c)}(x, \theta) \gamma_{\mu} \Psi^{(a c)}(x, \theta)=J_{\mu}(x)+\theta\left(s_{a b} J_{\mu}(x)\right) \\
& \equiv J_{\mu}(x)+i \theta\left(\bar{b}_{2} \gamma_{\mu} \psi-\bar{\psi} \gamma_{\mu} \bar{b}_{1}\right) .
\end{aligned}
$$

Exploiting the explicit nilpotent transformations $s_{a b}$ from (2.3), it can be checked that $s_{a b} J_{\mu}(x)=0$. Taking this as an input, the natural restriction on the super sub-manifold $\tilde{J}_{\mu}^{(a c)}(x, \theta)=J_{\mu}(x)$ implies the following condition

$$
\bar{b}_{2}(x) \gamma_{\mu} \psi(x)=\bar{\psi}(x) \gamma_{\mu} \bar{b}_{1}(x)
$$

As explained earlier in gory detail, it is clear that the above equality can be satisfied if one chooses $\bar{b}_{1}=-e \bar{C} \psi$ and $\bar{b}_{2}=-e \bar{\psi} \bar{C}$. Substituting these values in the expansion (4.7), we obtain the following expansion in the language of transformations $s_{a b}$ of (2.3):

$$
\Psi^{(a c)}(x, \theta)=\psi(x)+\theta\left(s_{a b} \psi(x)\right), \quad \bar{\Psi}^{(a c)}(x, \theta)=\bar{\psi}(x)+i \theta\left(s_{a b} \bar{\psi}(x)\right) .
$$

The above equation does establish the geometrical interpretation for the nilpotent charge $Q_{a b}$ (and the corresponding transformation $s_{a b}$ ) as the translation generator $(\partial / \partial \theta)$ for the anti-chiral superfields $\Psi^{(a c)}(x, \theta)$ and $\bar{\Psi}^{(a c)}(x, \theta)$ along the $\theta$-direction of the anti-chiral super sub-manifold. The nilpotency of the charge $Q_{a b}$ (and the corresponding transformation $s_{a b}$ ) is encoded in the two successive translations $(\partial / \partial \theta)^{2}=0$. However, we are not able to infer any geometrical interpretation for the anti-commutativity $s_{b} s_{a b}+s_{a b} s_{b}=0$ of the (anti-)BRST symmetry transformations (as well as the corresponding charges).

To dwell a bit on the geometrical origin of the anti-commutativity $s_{b} s_{a b}+s_{a b} s_{b}=0$ of the transformations (and their generators $Q_{b} Q_{a b}+Q_{a b} Q_{b}=0$ ) in the framework of the general augmented superfield formulation, we focus on the most general expansion in (4.1). One can construct the supercurrent $\tilde{J}_{\mu}(x, \theta, \bar{\theta})$ from the expansion (4.1) for the superfields with the following general super expansion

$$
\tilde{J}_{\mu}(x, \theta, \bar{\theta})=\bar{\Psi}(x, \theta, \bar{\theta}) \gamma_{\mu} \Psi(x, \theta, \bar{\theta})=J_{\mu}(x)+\theta \bar{K}_{\mu}(x)+\bar{\theta} K_{\mu}(x)+i \theta \bar{\theta} L_{\mu}(x),
$$

where the components $\bar{K}_{\mu}, K_{\mu}, L_{\mu}, J_{\mu}$ can be expressed in terms of the components of the basic super expansions (4.1), as

$$
\begin{aligned}
& \bar{K}_{\mu}(x)=i\left(\bar{b}_{2} \gamma_{\mu} \psi-\bar{\psi} \gamma_{\mu} \bar{b}_{1}\right), \quad K_{\mu}(x)=i\left(b_{1} \gamma_{\mu} \psi-\bar{\psi} \gamma_{\mu} b_{2}\right), \\
& L_{\mu}(x)=\bar{f} \gamma_{\mu} \psi+\bar{\psi} \gamma_{\mu} f+i\left(\bar{b}_{2} \gamma_{\mu} b_{2}-b_{1} \gamma_{\mu} \bar{b}_{1}\right), \quad J_{\mu}(x)=\bar{\psi} \gamma_{\mu} \psi
\end{aligned}
$$


To be consistent with our earlier observation that the BRST transformations $\left(s_{b}\right)$ are equivalent to the translations (i.e. $\left.\operatorname{Lim}_{\theta \rightarrow 0}(\partial / \partial \bar{\theta})\right)$ along the $\bar{\theta}$-direction and the anti-BRST $\left(s_{a b}\right)$ transformations are equivalent to the translations (i.e. $\operatorname{Lim}_{\bar{\theta} \rightarrow 0}(\partial / \partial \theta)$ ) along the $\theta$-direction of the supermanifold, it is straightforward to re-express the expansion in (4.11) as

$$
\tilde{J}_{\mu}(x, \theta, \bar{\theta})=J_{\mu}(x)+\theta\left(s_{a b} J_{\mu}(x)\right)+\bar{\theta}\left(s_{b} J_{\mu}(x)\right)+\theta \bar{\theta}\left(s_{b} s_{a b} J_{\mu}(x)\right) .
$$

It can be checked explicitly that, under the (anti-)BRST transformations (2.3), the conserved current $J_{\mu}(x)$ remains invariant (i.e. $\left.s_{b} J_{\mu}(x)=s_{a b} J_{\mu}(x)=0\right)$. This statement, with the help of (4.11) and (4.12), can be mathematically expressed as

$$
b_{1} \gamma_{\mu} \psi=\bar{\psi} \gamma_{\mu} b_{2}, \quad \bar{b}_{2} \gamma_{\mu} \psi=\bar{\psi} \gamma_{\mu} \bar{b}_{1}, \quad \bar{f} \gamma_{\mu} \psi+\bar{\psi} \gamma_{\mu} f=i\left(b_{1} \gamma_{\mu} \bar{b}_{1}-\bar{b}_{2} \gamma_{\mu} b_{2}\right)
$$

One of the possible solutions of the above restrictions, in terms of the components of the basic expansions in (4.1) and the basic fields of the Lagrangian density (2.2), is

$$
\begin{aligned}
& b_{1}=-e \bar{\psi} C, \quad b_{2}=-e C \psi, \quad \bar{b}_{1}=-e \bar{C} \psi, \quad \bar{b}_{2}=-e \bar{\psi} \bar{C}, \\
& f=-i e[B+e \bar{C} C] \psi, \quad \bar{f}=+i e \bar{\psi}[B+e C \bar{C}] .
\end{aligned}
$$

The above solutions are the unique solutions to all the restrictions in (4.14). Ultimately, the natural restriction that emerges on the $(2+2)$-dimensional supermanifold is $\tilde{J}_{\mu}(x, \theta, \bar{\theta})=J_{\mu}(x)$. Physically, this mathematical equation implies that there is no superspace contribution to the ordinary conserved current $J_{\mu}(x)$. It is straightforward to check that the substitution of (4.15) into (4.1) leads to the following

$$
\begin{aligned}
& \Psi(x, \theta, \bar{\theta})=\psi(x)+\theta\left(s_{a b} \psi(x)\right)+\bar{\theta}\left(s_{b} \psi(x)\right)+\theta \bar{\theta}\left(s_{b} s_{a b} \psi(x)\right) \\
& \bar{\Psi}(x, \theta, \bar{\theta})=\bar{\psi}(x)+\theta\left(s_{a b} \bar{\psi}(x)\right)+\bar{\theta}\left(s_{b} \bar{\psi}(x)\right)+\theta \bar{\theta}\left(s_{b} s_{a b} \bar{\psi}(x)\right) .
\end{aligned}
$$

This establishes the fact that (i) the nilpotent (anti-)BRST charges $Q_{(a) b}$ are the translations generators $\left(\operatorname{Lim}_{\bar{\theta} \rightarrow 0}(\partial / \partial \theta)\right) \operatorname{Lim}_{\theta \rightarrow 0}(\partial / \partial \bar{\theta})$ along the $(\theta) \bar{\theta}$ directions of the supermanifold. (ii) The property of the nilpotency (i.e. $Q_{(a) b}^{2}=0$ ) is encoded in the two successive translations along the Grassmannian directions of the supermanifold (i.e. $\left.(\partial / \partial \theta)^{2}=(\partial / \partial \bar{\theta})^{2}=0\right)$. (iii) The anticommutativity $\left(Q_{b} Q_{a b}+Q_{a b} Q_{b}=0\right)$ property is encoded in $(\partial / \partial \bar{\theta})(\partial / \partial \theta)+(\partial / \partial \theta)(\partial / \partial \bar{\theta})=0$ which also implies $s_{b} s_{a b}+s_{a b} s_{b}=0$.

Now we shall concentrate on the derivation of the symmetry transformations (2.4) on the matter fields in the framework of augmented superfield formulation. To this end in mind, we construct the super $\gamma_{5}$-axial-vector current $\tilde{J}_{\mu}^{(5)}(x, \theta, \bar{\theta})$ and substitute (4.1) to obtain

$$
\begin{aligned}
\tilde{J}_{\mu}^{(5)}(x, \theta, \bar{\theta}) & =\bar{\Psi}(x, \theta, \bar{\theta}) \gamma_{\mu} \gamma_{5} \Psi(x, \theta, \bar{\theta}) \\
& =J_{\mu}^{(5)}(x)+\theta \bar{K}_{\mu}^{(5)}(x)+\bar{\theta} K_{\mu}^{(5)}(x)+i \theta \bar{\theta} L_{\mu}^{(5)}(x),
\end{aligned}
$$

where the above components on the r.h.s. can be expressed, in terms of the basic components of the expansion in (4.1), as

$$
\begin{aligned}
& \bar{K}_{\mu}^{(5)}(x)=i\left(\bar{b}_{2} \gamma_{\mu} \gamma_{5} \psi-\bar{\psi} \gamma_{\mu} \gamma_{5} \bar{b}_{1}\right), \quad K_{\mu}^{(5)}(x)=i\left(b_{1} \gamma_{\mu} \gamma_{5} \psi-\bar{\psi} \gamma_{\mu} \gamma_{5} b_{2}\right) \\
& L_{\mu}^{(5)}(x)=\bar{f} \gamma_{\mu} \gamma_{5} \psi+\bar{\psi} \gamma_{\mu} \gamma_{5} f+i\left(\bar{b}_{2} \gamma_{\mu} \gamma_{5} b_{2}-b_{1} \gamma_{\mu} \gamma_{5} \bar{b}_{1}\right), \quad J_{\mu}^{(5)}(x)=\bar{\psi} \gamma_{\mu} \gamma_{5} \psi
\end{aligned}
$$


Invoking the condition $\tilde{J}_{\mu}^{(5)}(x, \theta, \bar{\theta})=J_{\mu}^{(5)}(x)$, we obtain the following restrictions on the components of the super expansion in (4.17):

$$
K_{\mu}^{(5)}(x)=0, \quad \bar{K}_{\mu}^{(5)}(x)=0, \quad L_{\mu}^{(5)}(x)=0 .
$$

Ultimately, as discussed earlier in gory details, these conditions lead to

$$
\begin{aligned}
& b_{1}=+e \bar{\psi} \bar{C} \gamma_{5}, \quad b_{2}=-e \bar{C} \gamma_{5} \psi, \quad \bar{b}_{1}=-e C \gamma_{5} \psi, \quad \bar{b}_{2}=+e \bar{\psi} C \gamma_{5}, \\
& f=+i e\left[\mathcal{B} \gamma_{5}-e C \bar{C}\right] \psi, \quad \bar{f}=+i e \bar{\psi}\left[\mathcal{B} \gamma_{5}+e \bar{C} C\right] .
\end{aligned}
$$

The substitution of the above values in the super expansion in (4.1) leads to the analogous expansion as in (4.16) with the replacements: $s_{b} \rightarrow s_{d}, s_{a b} \rightarrow s_{a d}$. Thus, we obtain

$$
\begin{aligned}
& \Psi(x, \theta, \bar{\theta})=\psi(x)+\theta\left(s_{a d} \psi(x)\right)+\bar{\theta}\left(s_{d} \psi(x)\right)+\theta \bar{\theta}\left(s_{d} s_{a d} \psi(x)\right) \\
& \bar{\Psi}(x, \theta, \bar{\theta})=\bar{\psi}(x)+\theta\left(s_{a d} \bar{\psi}(x)\right)+\bar{\theta}\left(s_{d} \bar{\psi}(x)\right)+\theta \bar{\theta}\left(s_{d} s_{a d} \bar{\psi}(x)\right) .
\end{aligned}
$$

This provides the geometrical interpretation for the nilpotent (anti-)co-BRST charges as the translation generators along the $(\theta) \bar{\theta}$-directions of the supermanifold. The geometrical interpretation for the nilpotency and anticommutativity properties is found to be exactly identical to the case of (anti-)BRST charges (as discussed after (4.16)) .

It is very interesting to point out that the (anti-)co-BRST transformations of (4.21) can be derived separately and independently as well. For this purpose, first we take the chiral limit (i.e. $\theta \rightarrow 0$ ) of the most general expansion in (4.1) so that we can obtain the expansion in (4.2) for the chiral superfields $\Psi^{(c)}(x, \bar{\theta})$ and $\bar{\Psi}^{(c)}(x, \bar{\theta})$. We can construct a $\gamma_{5}$-chiral supercurrent in terms of the expansion in (4.2) as

$$
\tilde{J}_{\mu}^{(5 c)}(x, \bar{\theta})=\bar{\Psi}^{(c)}(x, \bar{\theta}) \gamma_{\mu} \gamma_{5} \Psi^{(c)}(x, \bar{\theta})=J_{\mu}^{(5)}(x)+i \bar{\theta}\left(b_{1} \gamma_{\mu} \gamma_{5} \psi-\bar{\psi} \gamma_{\mu} \gamma_{5} b_{2}\right)
$$

It is clear that the above expansion in (4.22) can be re-expressed, vis-à-vis (4.13), as

$$
\tilde{J}_{\mu}^{(5 c)}(x, \bar{\theta})=\bar{\Psi}^{(c)}(x, \bar{\theta}) \gamma_{\mu} \gamma_{5} \Psi^{(c)}(x, \bar{\theta})=J_{\mu}^{(5)}(x)+\bar{\theta}\left(s_{d} J_{\mu}^{(5)}(x)\right) .
$$

However, it is can be readily checked, using (2.4), that $s_{d} J_{\mu}^{(5)}(x)=0$. Thus, we obtain a natural restriction on the (super)manifolds as $\tilde{J}_{\mu}^{(5 c)}(x, \theta)=J_{\mu}^{(5)}(x)$. In the language of the expansion in (4.22), this restriction can be satisfied by the choices of the components $b_{1}$ and $b_{2}$ as $b_{1}=+e \bar{\psi} \bar{C} \gamma_{5}$ and $b_{2}=-e \bar{C} \gamma_{5} \psi$. Insertions of these values into the chiral expansion in (4.2) leads to the following expansion in the language of the transformations (2.4)

$$
\Psi^{(c)}(x, \bar{\theta})=\psi(x)+\bar{\theta}\left(s_{d} \psi(x)\right), \quad \bar{\Psi}^{(c)}(x, \bar{\theta})=\bar{\psi}(x)+\bar{\theta}\left(s_{d} \bar{\psi}(x)\right) .
$$

Taking the help of our generic expression in (2.10), it is clear that the conserved and nilpotent dual(co-)BRST charge and the corresponding transformation $s_{d}$ geometrically imply the translation of the chiral superfields $\Psi^{(c)}(x, \bar{\theta})$ and $\bar{\Psi}^{(c)}(x, \bar{\theta})$ along the $\bar{\theta}$-direction of the chiral super sub-manifold parametrized by even variables $x^{\mu}$ and odd variable $\bar{\theta}$. In a similar fashion, we can obtain the nilpotent anti-co-BRST transformations by taking into 
account the anti-chiral limit (i.e. $\bar{\theta} \rightarrow 0$ ) of the expansion in (4.1) and, thereby, obtain the expansion given in (4.7). The latter equation allows us to write the $\gamma_{5}$-anti-chiral supercurrent in the expanded form as given below

$$
\tilde{J}_{\mu}^{(5 a c)}(x, \theta)=\bar{\Psi}^{(a c)}(x, \theta) \gamma_{\mu} \gamma_{5} \Psi^{(a c)}(x, \theta)=J_{\mu}^{(5)}(x)+i \theta\left(\bar{b}_{2} \gamma_{\mu} \gamma_{5} \psi-\bar{\psi} \gamma_{\mu} \gamma_{5} \bar{b}_{1}\right) .
$$

On the other hand, the anti-chiral $(\bar{\theta} \rightarrow 0)$ limit of the expansion in (4.17) leads to the

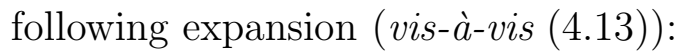

$$
\tilde{J}_{\mu}^{(5 a c)}(x, \theta, \bar{\theta})=\bar{\Psi}^{(a c)}(x, \theta) \gamma_{\mu} \gamma_{5} \Psi(x, \theta)=J_{\mu}^{(5)}(x)+\theta\left(s_{a d} J_{\mu}^{(5)}(x)\right) .
$$

Exploiting the explicit anti-co-BRST transformations of (2.4), it can be readily seen that $s_{s d} J_{\mu}^{(5)}(x)=0$. It is obvious that the consistency and conformity between (4.26) and (4.25) implies that $\bar{b}_{2} \gamma_{\mu} \gamma_{5} \psi=\bar{\psi} \gamma_{\mu} \gamma_{5} \bar{b}_{1}$. This restriction can be satisfied by the choice of $\bar{b}_{1}$ and $\bar{b}_{2}$ as $\bar{b}_{1}=-e C \gamma_{5} \psi, \bar{b}_{2}=+e \bar{\psi} C \gamma_{5}$. Plugging in these values in (4.7) leads to the following expansion for the anti-chiral superfields

$$
\Psi^{(a c)}(x, \theta)=\psi(x)+\theta\left(s_{a d} \psi(x)\right), \quad \bar{\Psi}^{(a c)}(x, \theta)=\bar{\psi}(x)+\theta\left(s_{a d} \bar{\psi}(x)\right) .
$$

The above equation provides the geometrical interpretation for the nilpotent anti-co-BRST charge $Q_{a d}$ and the corresponding nilpotent transformation $s_{a d}$ as the translational generator $(\partial / \partial \theta)$ for the anti-chiral superfields $\Psi^{(a c)}(x, \theta)$ and $\Psi^{(a c)}(x, \theta)$ along the $\theta$-direction of the anti-chiral three $(2+1)$-dimensional supermanifold parametrized by a couple of even variables $x^{\mu}(\mu=0,1)$ and an odd variable $\theta$. It is clear that the super translation $(\partial / \partial \theta)$ of the above anti-chiral superfields along the $\theta$-direction of the supermanifold generates the internal nilpotent symmetry transformations $s_{a d}$ on the ordinary fermionic Dirac fields $\psi(x)$ and $\bar{\psi}(x)$ located on the ordinary manifold, parametrized by $x^{\mu}$.

To wrap up this Section, we make a couple of general remarks on the discrete symmetry transformations in (2.11) as well as the nilpotent (anti-)BRST- and (anti-)co-BRST symmetry transformations (and their corresponding nilpotent generators). First, it can be checked that the supersymmetric counterpart of the discrete transformations (2.11) are

$$
\begin{aligned}
& C \rightarrow \pm i \gamma_{5} \bar{C}, \quad \psi \rightarrow \psi, \quad \bar{\psi} \rightarrow \bar{\psi}, \quad A_{0} \rightarrow \pm i \gamma_{5} A_{1}, \quad A_{1} \rightarrow \pm i \gamma_{5} A_{0}, \\
& \bar{C} \rightarrow \pm i \gamma_{5} C, \quad e \rightarrow \mp i e, \quad \mathcal{B} \rightarrow \mp i \gamma_{5} B, \quad B \rightarrow \mp i \gamma_{5} \mathcal{B}, \\
& \theta \rightarrow \theta, \quad \bar{\theta} \rightarrow \bar{\theta}, \quad S_{0} \rightarrow \pm i \gamma_{5} S_{1}, \quad S_{1} \rightarrow \pm i \gamma_{5} S_{0}, \quad b_{1} \rightarrow b_{1}, \quad b_{2} \rightarrow b_{2}, \\
& R_{0} \rightarrow \pm i \gamma_{5} R_{1}, \quad \bar{R}_{0} \rightarrow \pm i \gamma_{5} \bar{R}_{1}, \quad R_{1} \rightarrow \pm i \gamma_{5} R_{0}, \quad \bar{R}_{1} \rightarrow \pm i \gamma_{5} \bar{R}_{0}, \\
& \bar{b}_{1} \rightarrow \bar{b}_{1}, \quad \bar{b}_{2} \rightarrow \bar{b}_{2}, \quad f \rightarrow f, \quad f \rightarrow \bar{f} .
\end{aligned}
$$

It is straightforward to check that, under the above discrete transformations, the superfields in (3.1) and (4.1) transform in analogous manner as their ordinary counterparts. These are

$$
\begin{aligned}
& B_{0} \rightarrow \pm i \gamma_{5} B_{1}, \quad B_{1} \rightarrow \pm i \gamma_{5} B_{0}, \quad \Phi \rightarrow \pm i \gamma_{5} \bar{\Phi} \\
& \bar{\Phi} \rightarrow \pm i \gamma_{5} \Phi, \quad \Psi \rightarrow \Psi, \quad \bar{\Psi} \rightarrow \bar{\Psi}
\end{aligned}
$$

The above transformations are the analogue of the $\star$ operations defined in $(3.15)$ for the superspace differentials and their wedge products. However, the operation of the $\star$, listed 
in the language of the discrete symmetry transformations in (4.29), is applicable in the space of superfields. To obtain the analogue of equation (2.12), we have to express the nilpotent (anti-)BRST and (anti-)co-BRST symmetry transformations in the language of the superfields. We have not achieved this goal in our present paper. We hope to come to it later. Second, the common geometrical interpretations for the nilpotent (anti-)BRSTand (anti-)co-BRST charges can be succinctly expressed in the mathematical form, using the general expression (2.10) for the transformations, as

$$
\begin{aligned}
& s_{p} \Omega(x)=\operatorname{Lim}_{\theta \rightarrow 0} \frac{\partial}{\partial \bar{\theta}} \Omega^{(s)}(x, \theta, \bar{\theta}) \equiv-i\left[\Omega(x), Q_{p}\right]_{ \pm}, \\
& s_{q} \Omega(x)=\operatorname{Lim}_{\bar{\theta} \rightarrow 0} \frac{\partial}{\partial \theta} \Omega^{(s)}(x, \theta, \bar{\theta}) \equiv-i\left[\Omega(x), Q_{q}\right]_{ \pm},
\end{aligned}
$$

where $p=b, d, \quad q=a b, a d$ and $\Omega(x)=\psi(x), \bar{\psi}(x), C(x), \bar{C}(x), A_{\mu}(x)$ and $\Omega^{(s)}(x, \theta, \bar{\theta})=$ $\Psi(x, \theta, \bar{\theta}), \bar{\Psi}(x, \theta, \bar{\theta}), \Phi(x, \theta, \bar{\theta}), \bar{\Phi}(x, \theta, \bar{\theta}), B_{\mu}(x, \theta, \bar{\theta})$. The $(+)-$ signs on the brackets correspond to the (anti-)commutators for the generic field $\Omega(x)$ being (fermionic)bosonic in nature. Thus, it is clear that the following mapping exists among the symmetry transformations, the conserved charges and the translation generators along the Grassmannian directions

$$
\begin{aligned}
s_{b} \leftrightarrow Q_{b} \leftrightarrow \operatorname{Lim}_{\theta \rightarrow 0} \frac{\partial}{\partial \bar{\theta}}, & s_{d} \leftrightarrow Q_{d} \leftrightarrow \operatorname{Lim}_{\theta \rightarrow 0} \frac{\partial}{\partial \bar{\theta}}, \\
s_{a d} \leftrightarrow Q_{a d} \leftrightarrow \operatorname{Lim}_{\bar{\theta} \rightarrow 0} \frac{\partial}{\partial \theta}, & s_{a b} \leftrightarrow Q_{a b} \leftrightarrow \operatorname{Lim}_{\bar{\theta} \rightarrow 0} \frac{\partial}{\partial \theta} .
\end{aligned}
$$

It will be noted that in equations (4.30) and (4.31), we have taken into account only the off-shell nilpotent symmetries and their corresponding generators. However, it is straightforward to express these kind of relations for the on-shell nilpotent symmetries and their corresponding generators as well.

\section{Conclusions}

The central theme of our present investigation was to provide the geometrical origin and interpretation for the off-shell as well as on-shell nilpotent symmetries (and their corresponding nilpotent generators) in the language of the translations along the Grassmannian directions of the supermanifold. These twin objectives have been achieved by exploiting the augmented superfield formulation for the case of the 2D QED considered on the general four $(2+2)$-dimensional supermanifold as well as its (anti-)chiral three $(2+1)$-dimensional super sub-manifolds, respectively. The key roles in our present endeavour are played by the (dual-)horizontality conditions, invariance of the matter (super)currents and the nilpotent super de Rham cohomological operators $\tilde{d}$ and $\tilde{\delta}=-\star \tilde{d} \star$ (and their ordinary counterparts $d$ and $\delta=-* d *$ ) on the (super)manifolds. In particular, the nilpotent cohomological operators are useful in (i) providing the basis for the (dual-)horizontality conditions imposed on the supermanifolds, (ii) providing the derivations of the nilpotent symmetries for the 
gauge- and (anti-)ghost fields of the interacting gauge theory (i.e. 2D QED), (iii) providing the geometrical interpretations for the nilpotent symmetries and their corresponding generators, and (iv) providing the origin for the existence of the kinetic energy and gauge-fixing terms of the Lagrangian density (cf.(2.1) and (2.2)) of the BRST invariant gauge theory.

As discussed in Section 2, the cohomological operators $(d, \delta, \Delta)$ find their physical interpretation in the language of the symmetry properties of the BRST invariant Lagrangian density (2.2) for the 2D QED. The invariance of the kinetic energy term (constructed by $d$ and $A$ ) is at the heart of the existence of the (anti-)BRST symmetry transformations. In contrast, the (anti-)co-BRST symmetry transformations owe their existence to the invariance of the gauge-fixing term (constructed by $\delta$ and $A$ ) of the Lagrangian density (2.2) of 2D QED. The analogue of the Laplacian operator $\Delta$ is a bosonic symmetry transformation (equal to the anticommutator of the nilpotent (anti-)BRST and (anti-)co-BRST symmetry transformations) under which the ghost term of the Lagrangian density (2.2) does not transform ${ }^{\ddagger}$. As a consequence, the generators of the above symmetry transformations $Q_{(a) b}, Q_{(a) d}$ and $W=\left\{Q_{b}, Q_{d}\right\}=\left\{Q_{a d}, Q_{a b}\right\}$ mathematically rely for their existence to the cohomological operators $d, \delta, \Delta$. In fact, the ghost number consideration allows us to obtain the following two-to-one mapping from the set of local and conserved charges to the set of the de Rham cohomological operators

$$
\left(Q_{b}, Q_{a d}\right) \rightarrow d, \quad\left(Q_{d}, Q_{a b}\right) \rightarrow \delta, \quad W=\left\{Q_{b}, Q_{d}\right\}=\left\{Q_{a d}, Q_{a b}\right\} \rightarrow \Delta
$$

Due to the above equation, any arbitrary state $\mid \phi>_{n}$ in the quantum Hilbert space, with the ghost number $n$ (i.e. $i Q_{g}\left|\phi>_{n}=n\right| \phi>_{n}$ ), can be decomposed into a harmonic state $\mid h>_{n}\left(\right.$ with $\left.Q_{b}\left|h>_{n}=Q_{d}\right| h>_{n}=0\right)$, a BRST exact state $Q_{b} \mid \theta>_{n-1}$ and a BRST co-exact state $Q_{d} \mid \chi>_{n+1}$ as given below $[27,35,36]$

$$
\left|\phi>_{n}=\right| h>_{n}+Q_{b}\left|\theta>_{n-1}+Q_{d}\right| \chi>_{n+1}=\left|h>_{n}+Q_{a d}\right| \theta>_{n-1}+Q_{a b} \mid \chi>_{n+1} .
$$

The above equation is the analogue of the Hodge decomposition theorem for a differential form (cf. the footnote on page 3) (see, e.g., [20-24] for details).

One of the key observations of our present investigation is the consistency and complementarity between the (dual-)horizontality conditions and the requirement of the invariance of the matter (super)currents on the (super)manifolds. It is obvious that the augmented superfield formulation is the generalization of the usual superfield formalism because (i) the geometrical interpretation for the nilpotent charges in the language of the translation generators, along the Grassmannian directions, remains intact, (ii) the nilpotency of the above conserved charges (as well as the cohomological operators) is found to be encoded in the property $(\partial / \partial \theta)^{2}=0,(\partial / \partial \bar{\theta})^{2}=0$. Geometrically, the above relations imply that two successive translations of any superfields along either of the two Grassmannian directions corresponds to no translations at all, (iii) the geometrical interpretation for the

$\ddagger$ The key role played by $\Delta$, however, is in the context of the equation of motion for the gauge field $A_{\mu}$ for the case of 2D QED which is described by the gauge-fixed Lagrangian density (2.2). 
anticommutativity of $s_{b} s_{a b}+s_{a b} s_{b}=0$ and $s_{d} s_{a d}+s_{a d} s_{d}=0$ is hidden in the relation $(\partial / \partial \theta)(\partial / \partial \bar{\theta})+(\partial / \partial \bar{\theta})(\partial / \partial \theta)=0$, (iv) the nilpotent transformations on the gauge- and (anti-)ghost fields derived from the (dual-)horizontality condition are found to be consistent with the analogous transformations on the matter fields derived due to the requirements of the invariance of the matter (super)currents on the (super)manifolds.

The consistency and complementarity between the restrictions due to (i) the horizontality condition, and (ii) the invariance of the conserved matter (super)current on the (super)manifolds can be understood in the language of the gauge invariance. It is known that, for the Abelian gauge theory, the 2-form curvature $F=d A$ remains invariant under the gauge (or BRST) transformation $A \rightarrow A+d C$ which involves the gauge- and ghost fields. This is why, the horizontality condition $\tilde{d} \tilde{A}=d A$ produces the nilpotent BRST transformations on the gauge- and ghost fields. In fact, the gauge (or BRST) transformations on the matter fields in (2.3) owe their origin to the gauge transformation $A \rightarrow A+d C$.

Thus, the requirement of the matter conserved (super)currents $\tilde{J}_{\mu}(x, \theta, \bar{\theta})=J_{\mu}(x)$ on the (super)manifolds yields the nilpotent BRST transformations on the matter fields that are found to be consistent with the nilpotent transformations on the gauge- and (anti-)ghost fields. In the language of the superfield formalism, there is a key difference between the derivation of the nilpotent symmetries for the gauge- as well as (anti-)ghost fields and the matter fields. Whereas the choice of the (anti-)chiral superfields yields the on-shell nilpotent symmetries for the gauge- and (anti-)ghost fields, the same choices do not produce a different kind of symmetry transformations for the matter fields. In other words, the choices of the (anti-)chiral superfields have no major effect on the transformations of the matter fields.

It would be very nice and challenging endeavour to extend our present work to the case of 2D interacting non-Abelian gauge theory where there is an interaction between the matter fields and the non-Abelian gauge field. In fact, the (anti-)BRST symmetries for all the fields present in the 4D BRST invariant Lagrangian density of the non-Abelian gauge theory have already been derived in the augmented superfield formalism [33]. However, the non-local, non-covariant, continuous and nilpotent (anti-)co-BRST symmetries that exist (see, e.g., [37,38,48,49] for details) for the 4D (non-)Abelian gauge theories have not yet been derived in this augmented superfield approach. These are some of the open problems which are under investigation and our results will be reported elsewhere [50].

\section{References}

[1] P. A. M. Dirac, Lectures on Quantum Mechanics, (Yeshiva University Press, New York, 1964). For a review, see, e.g., K. Sundermeyer Constrained Dynamics: Lecture Notes in Physics vol. 169 (Springer-Verlag, Berlin, 1982).

[2] For an extensive review, see, e.g., M. Henneaux and C. Teitelboim, Quantization of Gauge Systems (Princeton University Press, New Jersey, Princeton, 1992). 
[3] I. J. R. Aitchison and A. J. G. Hey, Gauge Theories in Particle Physics: A Practical Introduction (Adam Hilger, Bristol, 1982).

[4] K. Nishijima, Colour confinement based on BRS algebra, in: Progress in Quantum Field Theory Eds. H. Ezawa and S. Kamefuchi (North-Holland, Amsterdam, 1986) pp. 99-119

For an extensive review, see, e.g., K. Nishijima, BRS invariance, asymptotic freedom and colour confinement, Czech. J. Phys. 46 (1996) 1-124.

[5] A. S. Schwarz, The partition function of degenerate quadratic functional and RaySinger invariants, Lett. Math. Phys. 2 (1978) 247-252;

E. Witten, Topological quantum field theory,

Comm. Math. Phys. 117 (1988) 353-415;

E. Witten, Quantum field theory and Jones polynomial, Comm. Math. Phys. 121 (1989) 351-435.

[6] For a review, see, e.g., D. Birmingham, M. Blau, M. Rakowski and G. Thompson, Topological field theory, Phys. Rep. 209 (1991) 129-340.

[7] See, e.g., R. Gopakumar and C. Vafa, M-theory and topological strings-I, hep-th/9808187;

See, e.g., R. Gopakumar and C. Vafa, M-theory and topological strings-II, hep-th/9812127.

[8] I. A. Batalin and I. V. Tyutin, Existence theorem for the effective gauge algebra in the generalized canonical formalism with Abelian conversion of second class constraints, Int. J. Mod. Phys. A 6 (1991) 3255-3282.

[9] M. B. Green, J. H. Schwarz and E. Witten, Superstring Theory vols 1 and 2 (Cambridge University Press, Cambridge, 1987).

[10] J. Polchinski, String Theory, vols 1 and 2 (Cambridge University Press, Cambridge, 1998).

[11] J. Thierry-Mieg, Geometrical reinterpretation of Faddeev-Popov ghost particles and BRS transformations, J. Math. Phys. 21 (1980) 2834-2838.

[12] M. Quiros, F. J. de Urries, J. Hoyos, M. L. Mazon and E. Rodrigues, Geometrical structure of Faddeev-Popov fields and invariance properties of gauge theories, J. Math. Phys. 22 (1981) 1767-1790.

[13] R. Delbourgo and P. D. Jarvis, Extended BRS invariance and $O S p(4 \mid 2)$ supersymmetry, J. Phys. A: Math. Gen. 15 (1981) 611-625;

R. Delbourgo, P. D. Jarvis and G. Thompson, Local $O S p(4 \mid 2)$ supersymmetry and extended BRS transformations for gravity, Phys. Lett. B 109 (1982) 25-28.

[14] L. Bonora and M. Tonin, Suprfield formulation of extended BRS symmetry, Phys. Lett. B 98 (1981) 48-55;

L. Bonora, P. Pasti and M. Tonin, Geometric description of extended BRS symmetry in superfield formulation, Nuovo Cimento 63 A (1981) 353-366.

[15] L. Baulieu and J. Thierry-Mieg, The principle of BRS symmetry: an alternative approach to Yang-Mills theories, Nucl. Phys. B 197 (1982) 477-530;

L. Alvarez-Gaumé and L. Baulieu, The two quantum symmetries associated with a classical symmetry, Nucl. Phys. B 212 (1983) 255-270. 
[16] D. S. Hwang and C. -Y. Lee, Non-Abelian topological mass generation in fourdimensions, J. Math. Phys. 38 (1997) 30-38.

[17] P. M. Lavrov, P. Yu. Moshin and A. A. Rashetnyak, Superfield formulation of the Lagrangian BRST quantization method, Mod. Phys. Lett. A 10 (1995) 2687-2694;

P. M. Lavrov and P. Yu. Moshin, Superfield Lagrangian quantization with extended BRST symmetry, Phys. Lett. B 508 (2001) 127-136.

[18] N. R. F. Braga and A. Das, A superspace formulation of the BV action, Nucl. Phys. B 442 (1995) 655-668.

[19] N. Nakanishi and I. Ojima, Covariant Operator Formalism of Gauge Theories and Quantum Gravity (World Scientific, Singapore, 1990).

[20] T. Eguchi, P. B. Gilkey and A. J. Hanson, Gravitation, gauge theories and differential geometry, Phys. Rep. 66 (1980) 213-393.

[21] S. Mukhi and N. Mukunda, Introduction to Topology, Differential Geometry and Group Theory for Physicists (Wiley Eastern Pvt. Ltd., New Delhi, 1990).

[22] J. W. van Holten, The BRST complex and the cohomology of compact Lie algebras, Nucl. Phys. B 339 (1990) 158-176.

[23] K. Nishijima, The Casimir operator in the representations of BRS algebra, Prog. Theo. Phys. 80 (1988) 897-904;

K. Nishijima, Observable states in the representations of BRS algebra, Prog. Theo. Phys. 80 (1988) 905-911.

[24] H. Aratyn, BRS cohomology in string theory: geometry of Abelization and quartet mechanism, J. Math. Phys. 31 (1990) 1240-1258;

G. Fülöp and R. Marnelius, Gauge-fixing and co-BRST, Nucl. Phys. B 456 (1995) 442-472.

[25] R. P. Malik, New symmetries for Abelian gauge theory in superfield formulation, Phys. Lett. B 521 (2001) 409-417, hep-th/0108105.

[26] R. P. Malik, Superfield approach to BRST cohomology, J. Phys. A: Math. Gen. 35 (2002) 3711-3726, hep-th/01060215.

[27] R. P. Malik, Cohomological aspects of gauge theories: superfield formalism, Ann. Phys. (N.Y.) 307 (2003) 1-40, hep-th/0205135.

[28] R. P. Malik, Superfield approach to a novel symmetry for non-Abelian gauge theory, Mod. Phys. Lett. A 17 (2002) 185-196, hep-th/0111253.

[29] R. P. Malik, Topological aspects of Abelian gauge theory in superfield formulation, $J$. Phys. A: Math. Gen 35 (2002) 6919-6930, hep-th/0112260.

[30] R. P. Malik, Superfield approach to topological features of non-Abelian gauge theory, J. Phys. A: Math. Gen 35 (2002) 8817-8830, hep-th/0204015.

[31] R. P. Malik, Nilpotent symmetries for QED in superfield formalism, Phys. Lett. B 584 (2004) 210-219, hep-th/0311001.

[32] R. P. Malik, Superfield approach to symmetries for matter fields in Abelian gauge theories, J. Phys. A: Math Gen 37 (2004) 5261-5274, hep-th/0311193. 
[33] R. P. Malik, Nilpotent symmetries for matter fields in non-Abelian gauge theory: augmented superfield formalism, hep-th/0402005.

[34] R. P. Malik, Nilpotent symmetries for a free relativistic particle in augmented superfield formalism, hep-th/0402123.

[35] R. P. Malik, New local symmetry for QED in two dimensions, Mod. Phys. Lett. A 15 (2000) 2079-2086, hep-th/0003128.

[36] R. P. Malik, Dual BRST symmetry for QED, Mod. Phys. Lett. A 16 (2001) 477-488, hep-th/9711056.

[37] See, e.g., D. McMullan and M. Lavelle, Nonlocal symmetry for QED, Phys. Rev. Lett. 71 (1993) 3758-3761.

[38] See, e.g, V. O. Rivelles, The several guises of the BRST symmetry, Phys. Rev. D 53 (1996) 3247-3251.

[39] R. P. Malik, Cohomological aspects of Abelian gauge theory, J. Phys. A: Math. Gen. 33 (2000) 2437-2446, hep-th/9902146.

[40] R. P. Malik, BRST cohomology and Hodge decomposition theorem in Abelian gauge theory, Int. J. Mod. Phys. A 15 (2000) 1685-1705, hep-th/9808040.

[41] R. P. Malik, New topological field theories in two dimensions, J. Phys. A: Math. Gen. 34 (2001) 4167-4182, hep-th/0012085.

[42] R. P. Malik, Topological aspects in non-Abelian gauge theory, Mod. Phys. Lett. A 14 (1999) 1937-1950, hep-th/9903121.

[43] E. Harikumar, R. P. Malik and M. Sivakumar, Hodge decomposition theorem for Abelian two form gauge theory, J. Phys. A: Math. Gen. 33 (2000) 7149-7164, hepth/0004145.

[44] R. P. Malik, Abelian 2-form gauge theory: special features, J. Phys. A: Math. Gen. 36 (2003) 5095-5114, hep-th/0209136.

[45] D. M. Gitman and I. V. Tyutin, Quantization of Fields with Constraints (Springer, Berlin, 1990).

[46] S. Weinberg, The Quantum Theory of Fields: Modern Applications vol. 2 (Cambridge University Press, Cambridge, 1996).

[47] S. Deser, A. Gomberoff, M. Henneaux and C. Teitelboim, Duality, self-duality, sources and charge quantization in Abelian n-form theories, Phys. Lett. B 400 (1997) 80-86.

[48] R. P. Malik, Superfield approach to (non-)local symmetries for 1-form Abelian gauge theory, J. Phys. A: Math. Gen. 37 (2004) 1059-1078, hep-th/0306182.

[49] R. P. Malik, Wigner's little group and BRST cohomology for one-form Abelian gauge theory, Int. J. Mod. Phys. A 19 (2004) 2721-2738, hep-th/0305118,

[50] R. P. Malik, in preparation. 